\title{
Time Varying Structural Vector Autoregressions and Monetary Policy
}

\author{
Giorgio E. Primiceri* \\ Northwestern University
}

First draft: April 2002

This version: July 2004

\begin{abstract}
Monetary policy and the private sector behavior of the US economy are modeled as a time varying structural vector autoregression, where the sources of time variation are both the coefficients and the variance covariance matrix of the innovations. The paper develops a new, simple modeling strategy for the law of motion of the variance covariance matrix and proposes an efficient Markov chain Monte Carlo algorithm for the model likelihood/posterior numerical evaluation. The main empirical conclusions are: 1) both systematic and non-systematic monetary policy have changed during the last forty years. In particular, systematic responses of the interest rate to inflation and unemployment exhibit a trend toward a more aggressive behavior, despite remarkable oscillations; 2) this has had a negligible effect on the rest of the economy. The role played by exogenous non-policy shocks seems more important than interest rate policy in explaining the high inflation and unemployment episodes in recent US economic history.
\end{abstract}

JEL Classification: C15, C22, E31, E32, E58.

Keywords: time varying coefficients, multivariate stochastic volatility, Gibbs sampling, systematic monetary policy, monetary policy shocks, identification.

${ }^{*}$ This paper is a revised version of the first chapter of my Ph.D. dissertation at Princeton University. I am indebted to Chris Sims, whose suggestions considerably improved the quality of the paper. I am also grateful to Timothy Cogley, Giovanni Favara, Paolo Giordani, Hidehiko Ichimura, Alejandro Justiniano, Siem Jan Koopman, Michele Lenza, Guido Lorenzoni, Bernard Salanié, Paolo Surico, Lars Svensson, Andrea Tambalotti, Thijs van Rens, especially Mark Watson and two anonymous referees for many helpful comments and discussions. Remaining errors are my own.

Department of Economics, Northwestern University, 2001 Sheridan Road, Evanston, IL 60208, USA. Email: g-primiceri@northwestern.edu. URL: http://www.faculty.econ.northwestern.edu/faculty/primiceri. 


\section{Introduction}

There is strong evidence that US unemployment and inflation were higher and more volatile in the period between 1965 and 1980 than in the last twenty years. The literature has considered two main classes of explanations for this difference in performance. The first class of explanations (see, for instance, Blanchard and Simon, 2001, Stock and Watson, 2002, Sims and Zha, 2004) focuses on the heteroskedasticity of the exogenous shocks, which have been much more volatile in the 70s and early 80 s than in the rest of the sample. The second class of explanations emphasizes the changes in the transmission mechanism, i.e. the way macroeconomic variables respond to shocks. Particular attention has been given to monetary policy. If monetary policy varies over time, this has a potential direct effect on the propagation mechanism of the innovations. Furthermore, if agents are rational and forward-looking, policy changes will be incorporated in the private sector's forecasts, inducing additional modifications in the transmission mechanism.

Many authors (among others Boivin and Giannoni, 2003, Clarida, Galí and Gertler, 2000, Cogley and Sargent, 2001 and 2003, Judd and Rudebusch, 1998, Lubik and Schorfheide, 2004) have argued that US monetary policy was less active against inflationary pressures under the Fed chairmanship of Arthur Burns than under Paul Volcker and Alan Greenspan. However, this view is controversial. Other studies have in fact found either little evidence of changes in the systematic part of monetary policy (Bernanke and Mihov, 1998, Hanson, 2003, Leeper and Zha, 2002) or no evidence of unidirectional drifts in policy toward a more active behavior (Sims, 1999 and 2001a).

This paper investigates the potential causes of the poor economic performance of the 70s and early 80s and to what extent monetary policy played an important role in these high unemployment and inflation episodes. The objective here is to provide a flexible framework for the estimation and interpretation of time variation in the systematic and non-systematic part of monetary policy and their effect on the rest of the economy. Two are the main characteristics required for an econometric framework able to address the issue: 1) time varying parameters in order to measure policy changes and implied shifts in the private sector behavior; 2) a multiple equation model of the economy in order to understand how changes in policy have affected the rest of the economy. For this purpose, this paper estimates a time varying structural vector autoregression (VAR), where the time variation derives both from the coefficients and the variance covariance matrix of the model's innovations. Notice that any reasonable attempt to model changes in policy, structure and their interaction must include time variation of the variance covariance matrix of the innovations. This reflects both time variation of the simultaneous relations among the variables of the model and heteroskedasticity of

the innovations. This is done by developing a simple multivariate stochastic volatility modeling strategy for the law of motion of the variance covariance matrix. The estimation of this model with drifting coefficients and multivariate stochastic volatility requires numerical methods. An efficient Markov chain Monte Carlo algorithm is proposed for the numerical evaluation of the posterior of the parameters of interest.

The methodology developed in the paper is used to estimate a small model of the US economy, 
delivering many empirical conclusions. First of all, there is evidence of changes both in nonsystematic and systematic monetary policy during the last forty years. The relative importance of non-systematic policy was significantly higher in the first part of the sample, suggesting that a Taylor-type rule is much less representative of the US monetary policy in the 60s and 70s than in the last fifteen years. Furthermore, private sector responses to non-systematic policy (monetary policy shocks) appear linear in the amplitude of non-systematic policy actions. Turning to the systematic part of policy, there is some evidence of higher interest rate responses to inflation and unemployment in the Greenspan period. However, a counterfactual simulation exercise suggests these changes did not play an important role in the high inflation and unemployment episodes in recent US economic history. In fact, the high volatility of the exogenous non-policy shocks seems to explain a larger fraction of the outbursts of inflation and unemployment of the 70 s and early 80 s.

From the methodological perspective, this paper is related to the fairly well developed literature on modeling and estimating time variation in multivariate linear structures. Canova (1993), Sims (1993), Stock and Watson (1996) and Cogley and Sargent (2001) model and estimate VARs with drifting coefficients. On the other hand, multivariate stochastic volatility models are discussed by Harvey, Ruiz and Shephard (1994), Jacquier, Polson and Rossi (1995), Kim, Shephard and Chib (1998), Chib, Nardari and Shephard (2002). However, these studies impose some restrictions on the evolution over time of the elements of the variance covariance matrix. Typical restrictions are either the assumption that the covariances do not evolve independently of the variances or a factor structure for the covariance matrix. Following this line of research, Cogley (2003) and Cogley and Sargent (2003) use time varying variances in the context of VARs with drifting coefficients. However, in their model the simultaneous relations among variables are time invariant. As it will be made clear in the next section, their analysis is limited to reduced form models, usable almost only for data description and forecasting. Boivin (2001) considers the opposite case of time varying simultaneous relations, but neglects the potential heteroskedasticity of the innovations. Ciccarelli and Rebucci (2003) extend the framework of Boivin (2001) allowing for t-distributed errors, which account for non-persistent changes in the scale of the variances over time. Uhlig (1997) introduces unrestricted multivariate stochastic volatility in the context of VARs, but his model assumes that the VAR coefficients are constant. Here instead, both the coefficients and the entire variance covariance matrix of the shocks are allowed to vary over time. This is crucial if the objective is distinguishing between changes in the typical size of the exogenous innovations and changes in the transmission mechanism.

There is also a more recent literature that models time variation in linear structures with discrete breaks, meant to capture a finite number of switching regimes (see, for instance, Hamilton, 1989, Kim and Nelson, 1999, Sims, 1999 and 2001a and Sims and Zha, 2004). Discrete breaks models may well describe some of the rapid shifts in policy. However they seem less suitable to capture changes in private sector behavior, where aggregation among agents usually plays the role of smoothing most of the changes. Furthermore, even in a structural VAR, the private sector equations can 
be considered as reduced form relations with respect to a possible underlying behavioral model, where policy and private sector behavior are not easily distinguishable. If policy responds also to expectational future variables (instead of only to current and past ones), then also the policy equation in the VAR will be a mixture of policy and private sector behavior, determining smoother changes of the coefficients. Finally, the existence of any type of learning dynamics by private agents or the monetary authorities definitely favors a model with smooth and continuous drifting coefficients over a model with discrete breaks.

From the perspective of the empirical application, this paper is related to a large literature that analyzes changes in the conduct of monetary policy and their effect on the rest of the economy. Most of the existing academic work has emphasized the role of monetary policy in the poor economic performance of the 70s (among others, see Judd and Rudebusch, 1998, Clarida, Galí and Gertler, 2000, Boivin, 2001, Cogley and Sargent, 2001 and 2003, Lubik and Schorfheide, 2004, Boivin and Giannoni, 2003, Favero and Rovelli, 2003). This paper contrasts the most popular view and stresses the role of heteroskedastic non-policy innovations. In this respect, the conclusions are more similar to Bernanke and Mihov (1998) and Sims and Zha (2004).

The paper is organized as follows: section 2 presents the time varying structural VAR model adopted in the paper. Section 3 illustrates the key steps of the estimation methodology. Section 4 discusses the empirical results of the application to the US economy. Section 5 concludes.

\section{The Model}

The model presented in this paper is a multivariate time series model with both time varying coefficients and time varying variance covariance matrix of the additive innovations. The drifting coefficients are meant to capture possible nonlinearities or time variation in the lag structure of the model. The multivariate stochastic volatility is meant to capture possible heteroskedasticity of the shocks and nonlinearities in the simultaneous relations among the variables of the model. Allowing for time variation both in the coefficients and the variance covariance matrix, leaves it up to the data to determine whether the time variation of the linear structure derives from changes in the size of the shocks (impulse) or from changes in the propagation mechanism (response).

It is worth noting that the model admits many types of shocks. Nevertheless the heteroskedasticity assumption is limited to the additive innovations. This is not only for reasons of convenience, but also because the time variation in the size of the additive shocks is a feature of many empirical applications in macroeconomics. ${ }^{1}$ Furthermore, as recently emphasized by Cogley and Sargent (2003), overlooking heteroskedasticity would generate fictitious dynamics in the random coefficients.

Consider the model

$$
y_{t}=c_{t}+B_{1, t} y_{t-1}+\ldots+B_{k, t} y_{t-k}+u_{t} \quad t=1, \ldots, T .
$$

$y_{t}$ is an $n \times 1$ vector of observed endogenous variables; $c_{t}$ is an $n \times 1$ vector of time varying coefficients

\footnotetext{
${ }^{1}$ See for instance Bernanke and Mihov (1998) or Sims and Zha (2004).
} 
that multiply constant terms; $B_{i, t}, i=1, \ldots, k$, are $n \times n$ matrices of time varying coefficients; $u_{t}$ are heteroskedastic unobservable shocks with variance covariance matrix $\Omega_{t}$. Without loss of generality, consider the triangular reduction of $\Omega_{t}$, defined by

$$
A_{t} \Omega_{t} A_{t}^{\prime}=\Sigma_{t} \Sigma_{t}^{\prime}
$$

where $A_{t}$ is the lower triangular matrix

$$
A_{t}=\left[\begin{array}{cccc}
1 & 0 & \cdots & 0 \\
\alpha_{21, t} & 1 & \ddots & \vdots \\
\vdots & \ddots & \ddots & 0 \\
\alpha_{n 1, t} & \cdots & \alpha_{n n-1, t} & 1
\end{array}\right]
$$

and $\Sigma_{t}$ is the diagonal matrix

$$
\Sigma_{t}=\left[\begin{array}{cccc}
\sigma_{1, t} & 0 & \cdots & 0 \\
0 & \sigma_{2, t} & \ddots & \vdots \\
\vdots & \ddots & \ddots & 0 \\
0 & \cdots & 0 & \sigma_{n, t}
\end{array}\right] .
$$

It follows that

$$
\begin{aligned}
y_{t} & =c_{t}+B_{1, t} y_{t-1}+\ldots+B_{k, t} y_{t-k}+A_{t}^{-1} \Sigma_{t} \varepsilon_{t}, \\
V\left(\varepsilon_{t}\right) & =I_{n} .
\end{aligned}
$$

Stacking in a vector $B_{t}$ all the right hand side coefficients, (3) can be rewritten as

$$
\begin{aligned}
y_{t} & =X_{t}^{\prime} B_{t}+A_{t}^{-1} \Sigma_{t} \varepsilon_{t}, \\
X_{t}^{\prime} & =I_{n} \otimes\left[1, y_{t-1}^{\prime}, \ldots, y_{t-k}^{\prime}\right],
\end{aligned}
$$

where the symbol $\otimes$ denotes the Kronecker product.

A decomposition of the variance covariance matrix resulting in (4) is common, especially in the literature considering the problem of efficiently estimating covariance matrices (see, for instance, Pinheiro and Bates, 1996, Pourahamadi, 1999 and 2000, Smith and Kohn, 2002). In the context of time varying VAR models, Cogley (2003) and Cogley and Sargent (2003) have a similar decomposition, but with a time invariant $A_{t}$ matrix. It is important to notice that allowing the matrix $A_{t}$ to vary over time is crucial for a time varying structural VAR. A constant $A_{t}$ would imply that an innovation to the $i$-th variable has a time invariant effect on the $j$-th variable. This is clearly undesirable if the objective is modeling time variation in a simultaneous equation model, where simultaneous interactions among variables are fundamental.

The modeling strategy consists of modeling the coefficient processes in (4) instead of (1). Observe that there is a one to one mapping between (1) and (4) that fully justifies this approach. Let 
$\alpha_{t}$ be the vector of non-zero and non-one elements of the matrix $A_{t}$ (stacked by rows) and $\sigma_{t}$ be the vector of the diagonal elements of the matrix $\Sigma_{t}$. The dynamics of the model's time varying parameters is specified as follows:

$$
\begin{aligned}
B_{t} & =B_{t-1}+\nu_{t} \\
\alpha_{t} & =\alpha_{t-1}+\zeta_{t} \\
\log \sigma_{t} & =\log \sigma_{t-1}+\eta_{t}
\end{aligned}
$$

where the distributional assumptions regarding $\left(\varepsilon_{t}, \nu_{t}, \zeta_{t}, \eta_{t}\right)$ are stated below. The elements of the vector $B_{t}$ are modeled as random walks, as well as the free elements of the matrix $A_{t}$. The standard deviations $\left(\sigma_{t}\right)$ are assumed to evolve as geometric random walks, belonging to the class of models known as stochastic volatility. It constitutes an alternative to ARCH models. The crucial difference is that the variances generated by (7) are unobservable components. ${ }^{2}$

It is well known that a random walk process hits any upper or lower bound with probability one and this is clearly an undesirable implication of this model. On the other hand, as long as (5), (6) and (7) are thought to be in place for a finite period of time and not forever, this set of assumptions should be innocuous. Moreover, the random walk assumption presents the advantages of focusing on permanent shifts and reducing the number of parameters in the estimation procedure. Notice that, in principle, the model can be easily extended to consider more general autoregressive processes. $^{3}$ Especially for the parameters of the variance covariance matrix, this constitutes an advantage of this model over the so called local scale models (see, for example, Shephard, 1994a and the multivariate generalization of Uhlig, 1997).

All the innovations in the model are assumed to be jointly normally distributed with the following assumptions on the variance covariance matrix:

$$
V=\operatorname{Var}\left(\left[\begin{array}{c}
\varepsilon_{t} \\
\nu_{t} \\
\zeta_{t} \\
\eta_{t}
\end{array}\right]\right)=\left[\begin{array}{cccc}
I_{n} & 0 & 0 & 0 \\
0 & Q & 0 & 0 \\
0 & 0 & S & 0 \\
0 & 0 & 0 & W
\end{array}\right],
$$

where $I_{n}$ is a $n$ dimensional identity matrix, $Q, S$ and $W$ are positive definite matrices. It is worth noting that none of the restrictions on the structure of $V$ are essential. All the zero blocks could be substituted by non-zero blocks, with only small modifications of the estimation procedure that will be described in the next section. Nevertheless, there are at least two reasons suggesting a choice of $V$ as the one described in (8). The first one is related to the already high number of parameters of the model. Adding all the off diagonal elements of $V$ would require the specification of a sensible prior, able to prevent cases of ill-determined parameters. The second (and more important) reason is that allowing for a completely generic correlation structure among different sources of uncertainty

\footnotetext{
${ }^{2}$ See Shephard (1996) for an overview of the econometric properties of the stochastic volatility model and a comparison with ARCH.

${ }^{3}$ Section 4.4 will provide some further discussion and evidence.
} 
would preclude any structural interpretation of the innovations. A complete discussion of the issue is postponed to the next section.

Most of the paper will adopt the additional assumption of $S$ being block diagonal, with blocks corresponding to parameters belonging to separate equations. In other words, the coefficients of the contemporaneous relations among variables are assumed to evolve independently in each equation. Even though it is not crucial, this assumption simplifies the inference and increases the efficiency of the estimation algorithm. The general case of $S$ unrestricted will be considered in section 4.4, which shows the robustness of the empirical results to this simplifying assumption.

\section{Bayesian Inference}

The following notation is used to denote the history of a generic vector of variables $\omega_{t}$ up to a generic time $\tau$ :

$$
\omega^{\tau}=\left[\omega_{1}^{\prime}, \ldots, \omega_{\tau}^{\prime}\right]^{\prime}
$$

For a generic matrix of variables and constant terms $M_{t}$,

$$
M^{\tau}=\left[m_{1}^{\prime}, \ldots, m_{\tau}^{\prime}\right]^{\prime}
$$

where $m_{t}$ is a column vector constructed with the time varying elements of $M_{t}$.

The objective of this section is to lay out the econometric techniques used to estimate the time varying structural VAR model presented in the previous section. Bayesian methods are used to evaluate the posterior distributions of the parameters of interest, i.e. the unobservable states, $B^{T}$, $A^{T}, \Sigma^{T}$ and the hyperparameters of the variance covariance matrix $V$. Observe that, dealing with unobservable components, where the distinction between parameters and shocks is less clear than in other situations, a Bayesian approach is the natural one. Three other reasons make Bayesian methods particularly suitable to estimate this class of models and preferable to classical estimation. First, if the variance of the time varying coefficients is small, the classical maximum likelihood estimator of this variance has a point mass at zero. This is related to the so called pile-up problem (see, for instance, Sargan and Bhargava, 1983, Shephard and Harvey, 1990, Stock and Watson, 1998). The second drawback of classical maximum likelihood is related to the high dimensionality and nonlinearity of the problem. Such a complicated model will quite possibly have a likelihood with multiple peaks, some of which in uninteresting or implausible regions of the parameter space. Moreover, if these peaks are very narrow, the likelihood may reach particularly high values, not at all representative of the model's fit on a wider and more interesting parameter region. In a Bayesian setting, the use of uninformative priors on reasonable regions of the parameter space is nevertheless effective in ruling out these misbehaviors. The third reason is practical: even though it is in principle possible to write up the likelihood of the model, it is a hard task to maximize it over such a high dimensional space. Bayesian methods deal efficiently with the high dimension of

the parameter space and the nonlinearities of the model, splitting the original estimation problem 
in smaller and simpler ones. Here, Gibbs sampling is used for the posterior numerical evaluation of the parameters of interest. Gibbs sampling is a particular variant of Markov chain Monte Carlo (MCMC) methods that consists of drawing from lower dimensional conditional posteriors as opposed to the high dimensional joint posterior of the whole parameter set.

Finally, observe that MCMC is a smoothing method and therefore delivers smoothed estimates, i.e. estimates of the parameters of interest based on the entire available set of data. The suitability of smoothed estimates, as opposed to filtered ones, cannot be established a priori, but clearly depends on the specific problem at hand. For example, filtered estimates are more appropriate if the objective of the investigation is constructing model diagnostics (and marginal likelihoods) or forecasting evaluation. The use of simulation based filters is required in these cases. Simulation based filters for these kind of problems are known as particle filters, which are beyond the scope of this paper (for an overview, see Doucet, De Freitas and Gordon, 2001). On the other hand, smoothed estimates are more efficient (and therefore preferable) when, like in this paper, the objective is an investigation of the true evolution of the unobservable states over time. In these cases, filtered estimates are inappropriate because they would exhibit transient variation even in time invariant models (as pointed out by Sims, 2001b).

The rest of the section contains a description of the priors, a sketch of the estimation strategy (the details are in appendix A) and an explanation of how to deal with non-triangular identification structures.

\subsection{Priors and Ordering}

The prior distributions proposed in this paper are chosen because of their intuitiveness and convenience in the applications. First of all, it is convenient to assume that the initial states for the coefficients, for the covariances, for the log volatilities and the hyperparameters are independent of each other. The priors for the hyperparameters, $Q, W$ and the blocks of $S$, are assumed to be distributed as independent inverse-Wishart. The priors for the initial states of the time varying coefficients, simultaneous relations and $\log$ standard errors, $p\left(B_{0}\right), p\left(\alpha_{0}\right)$ and $p\left(\log \sigma_{0}\right)$, are assumed to be normally distributed. ${ }^{4}$ These assumptions together with (5), (6) and (7) imply normal priors on the entire sequences of the $B$ 's, $\alpha$ 's and $\log \sigma$ 's (conditional on $Q, W$ and $S$ ).

The normal prior on $B$ is standard. Smith and Kohn (2002) use the same decomposition as in (2) and place a similar, normal prior on the elements of $A$. In the context of VAR models, Sims and Zha (1998) also use a similar prior. Placing a prior on the elements of $A$, as opposed to $A^{-1}$, might seem strange. However, the statistics literature has long recognized the advantages of modeling the inverse of the variance covariance matrix, as opposed to the covariance matrix itself (Dempster, 1972, Cox and Wermuth, 1996). The lognormal prior on the $\sigma$ 's is common in the stochastic volatility literature (see, for instance, Harvey, Ruiz and Shephard 1994, Kim, Shephard and Chib, 1998). Such a prior is not conjugate, but has the advantage of maintaining tractability.

\footnotetext{
${ }^{4}$ The values of means, variances, degrees of freedom and scale matrices are specified in the next section, where the application to the US data is discussed.
} 
On the other hand, inverse-gamma and inverse-Wishart priors (which are conjugate) have been used for local scale models (Shephard, 1994a and Uhlig, 1997). See Shephard (1994b) for a comparison of the two approaches in the univariate framework.

Finally, it is important to highlight a potential drawback of the modeling assumptions (4), (6), (7) and (8), in combination with the priors on the $A$ 's and $\Sigma$ 's. The drawback is the fact that, in principle, the order of the variables matters in (4). This is due to the lower triangular structure imposed on the $A_{t}$ matrix. More precisely, consider the vector $\tilde{y}_{t}$, obtained from a permutation of the order of the elements of the vector $y_{t}$. Similarly, let $\tilde{\Omega}_{t}$ denote the covariance matrix obtained from the same permutation of the rows and columns of $\Omega_{t}$. It can be shown that it is impossible to find a lower triangular matrix $\tilde{A}_{t}$ and a diagonal matrix $\tilde{\Sigma}_{t}$ such that

$$
\tilde{A}_{t}^{-1} \tilde{\Sigma}_{t} \tilde{\Sigma}_{t}^{\prime} \tilde{A}_{t}^{-1 \prime}=\tilde{\Omega}_{t}^{*}
$$

where the free elements of $\tilde{A}_{t}$ are normal, the free elements of $\tilde{\Sigma}_{t}$ are lognormal and $\tilde{\Omega}_{t}^{*}$ has the same distribution as $\tilde{\Omega}_{t}$. One way to see it is by noticing that the $(1,1)$ element of $\tilde{\Omega}_{t}^{*}$ would necessarily be lognormally distributed, while the $(1,1)$ element of $\tilde{\Omega}_{t}$ is not. ${ }^{5}$ Usually, in a time invariant setting, the lower triangular structure imposed on $A$ does not affect inference (because, for the variance covariance matrix, the likelihood soon dominates the prior). In this time varying setting however, it is less clear what may happen and the relevance of this issue might vary from case to case.

Observe that, if one is particularly concerned about this problem, there exists a natural solution, which is to impose a prior on all plausible orders of the variables of the system. Consequently, results obtained with different orders can be averaged on the basis of the prior or the posterior probabilities of different models (the models' posterior probabilities can be obtained, for instance, using the reversible jump MCMC method, described below). It is worth pointing out that, in the context of the empirical application of this paper, the results obtained with different orders of the variables are very similar.

\subsection{Simulation method}

The model is estimated by simulating the distribution of the parameters of interest, given the data. While the details are left to appendix A, this section sketches the MCMC algorithm used to generate a sample from the joint posterior of $\left(B^{T}, A^{T}, \Sigma^{T}, V\right)$. As mentioned above, Gibbs sampling is used in order to exploit the blocking structure of the unknowns. Gibbs sampling is carried out in four steps, drawing in turn time varying coefficients $\left(B^{T}\right)$, simultaneous relations $\left(A^{T}\right)$, volatilities $\left(\Sigma^{T}\right)$ and hyperparameters $(V)$, conditional on the observed data and the rest of the parameters.

Conditional on $A^{T}$ and $\Sigma^{T}$, the state space form given by (4) and (5) is linear and Gaussian. Therefore, the conditional posterior of $B^{T}$ is a product of Gaussian densities and $B^{T}$ can be drawn

\footnotetext{
${ }^{5}$ For example, in the two-dimensional case, the $(1,1)$ element of $\tilde{\Omega}_{t}$ would be the sum of a lognormal distribution and the product of another lognormal distribution and a normal distribution squared. This sum is not lognormal.
} 
using a standard simulation smoother (Fruhwirth-Schnatter, 1994 or Carter and Kohn, 1994). For the same reason, the posterior of $A^{T}$ conditional on $B^{T}$ and $\Sigma^{T}$ is also a product of normal distributions. Hence $A^{T}$ can be drawn in the same way. Drawing $\Sigma^{T}$ instead is more involved and relies mostly on the method presented in Kim, Shephard and Chib (1998). It consists of transforming a nonlinear and non-Gaussian state space form in a linear and approximately Gaussian one, which, again, allows the use of standard simulation smoothers. Simulating the conditional posterior of $V$ is standard, since it is the product of independent inverse-Wishart distributions.

Observe that the model and the posterior simulation method are close to Shephard (1994b). Indeed, the model belongs to the abstract class of partially non-Gaussian state space models, introduced by Shephard (1994b). However, this abstract class of models is quite broad and in Shephard (1994b) there is no treatment for the general multivariate case.

\subsection{Identification and structural interpretation}

The method described in the previous subsection and appendix A allows to estimate a reduced form VAR. However, as long as an exact identification scheme for the additive shocks is available, a structural VAR can be easily estimated in two steps. Consider the following structural VAR,

$$
y_{t}=X_{t}^{\prime} B_{t}+\Xi_{t} \varepsilon_{t}
$$

that differs from (4) because the $n \times n$ matrices $\Xi_{t}, t=1, \ldots, T$, are not necessarily lower triangular. Assume further that for any $t, \Xi_{t}$ contains at least $\frac{n(n-1)}{2}$ restrictions that guarantees identification. ${ }^{6}$ The first step consists of estimating the reduced form VAR, following the methodology illustrated in the previous sections. This first step delivers the posterior of the $B$ 's and the $\Omega^{\prime}{ }^{7}$ at every point in time. To obtain the numerical evaluation of the posterior of the $\Xi$ 's, it suffices to solve the system of equations given by

$$
\Xi_{t} \Xi_{t}^{\prime}=\Omega_{t}, \quad t=1, \ldots, T
$$

for every draw of $\Omega_{t}$. Observe that, even though this is a fairly general procedure, there may exist identifying structures of $\Xi_{t}$ for which (9) has no solution. As an illustrative example, consider the following structure for $\Xi_{t}$, in a 3 -by-3 case:

$$
\Xi_{t}=\left[\begin{array}{ccc}
\mathrm{x} & 0 & 0 \\
0 & \mathrm{x} & \mathrm{x} \\
\mathrm{x} & \mathrm{x} & \mathrm{x}
\end{array}\right],
$$

where the x's indicate the elements potentially different from zero. (10) implies a variance covariance matrix of the reduced form residuals with zero in the position $(1,2)$ that would not be compatible with draws of $\Omega_{t}$ obtained using the decomposition in (4) unless the element $(1,2)$ of the draw is

\footnotetext{
${ }^{6}$ These can be zero restrictions or cross elements restrictions.

${ }^{7}$ Remember that $\Omega_{t}, t=1, \ldots, T$, are the variance covariance matrices of the errors of the reduced form VAR at every point in time.
} 
very close to zero (in this case the solution would be approximate, but probably still reliable). If the draw of $\Omega_{t}$ has the $(1,2)$ element far from zero, the draw must be discarded. It is clear that the method is not efficient if the rejection rate is high. On the other hand, this is not too worrying if a high rejection rate is thought as a signal of a structure for $\Xi_{t}$ rejected by the data.

Clearly, if the identification is based on a triangular scheme (like the one in the next section), the solution to (9) is simply given by $\Xi_{t}=A_{t}^{-1} \Sigma_{t}$. Observe that this framework does not allow an efficient estimation of overidentified systems, unless the overidentification derives from further zero restrictions in a triangular scheme. This case is easily tractable by imposing the correspondent restrictions.

As anticipated in section 2, this is the right framework to attempt some structural interpretations. The fact that the elements of $\Xi$ are time varying represents the crucial difference between modeling time variation in a structural VAR as opposed to a standard VAR. In fact, models characterized by a time invariant variance covariance matrix (Sims, 1993, Canova, 1993 or Cogley and Sargent, 2001) or in which only the variances are drifting (Cogley, 2003, Cogley and Sargent, 2003) imply that all the contemporaneous relations among variables are time invariant. And this seems undesirable in a context in which all the lag coefficients are time varying. The other extreme, a model with time varying variance covariance matrix but constant coefficients (Uhlig, 1997), is probably less undesirable but may appear restrictive too.

Of course, the flexibility of the model of this paper does not come without costs. And the main one derives exactly from the lack of strong restrictions, able to isolate the sources of uncertainty. An example will clarify the issue: consider the common specification of the "monetary policy" VAR used below (a three variables VAR with inflation, unemployment and a short-term interest rate). Assume that a reliable identification scheme is available for the monetary policy equation (say a triangular identification based on the assumptions that inflation and unemployment react to the policy instrument with at least one period of lag). In a time invariant framework this would allow the use of standard structural VAR tools, as impulse responses and variance decompositions. This is because there is just one source of shocks for the monetary policy equation, that is the usual additive innovation. The situation is not as simple for a time varying structural VAR because the potential sources of monetary policy shocks are many and depend on the specific assumptions on the variance covariance matrix of all the innovations of the model. Looking at equation (8) for example, in the model described in this paper there would be at least 3 independent sources of monetary policy shocks. ${ }^{8}$ The first is the usual additive shock $(\varepsilon)$. The second is a shock to the interest rate reaction to current inflation and unemployment $(\zeta)$. The third is a shock to the interest rate reaction to past values of the variables of the system $(\nu)$. The first type of monetary policy shocks are assumed to be independent of any other innovation. This assumption is crucial for the interpretation of the effects of such shocks, besides the existence of other innovations in the system. On the other hand, the second type of shocks are assumed to be correlated within equation, but uncorrelated across equations (if $S$ is assumed block diagonal). This assumption would allow, for

\footnotetext{
${ }^{8}$ Not considering the shocks to the standard deviations of the additive innovations.
} 
example, the analysis of the impact of shocks to the systematic part of monetary policy on the rest of the economy. The third type of shocks are instead potentially correlated also across equations. This means that innovations of the third type to the non-policy equations will potentially affect also the monetary policy equation. However, this is not necessarily a bad assumption. On the contrary it seems to move in the direction of more realism in admitting that there exist sources of uncertainty in the system that cannot be easily separable and othogonalized for each equation. Actually, a full correlation structure for the block $\left[\zeta^{\prime}, \nu^{\prime}\right]$ would be even more appropriate, particularly if the VAR is thought as a reduced form representation of a forward-looking model, where most of the coefficients of the VAR would be convolutions of the original parameters of the model. However, while it is relatively easy to allow for a full variance covariance matrix for the block $\left[\zeta^{\prime}, \nu^{\prime}\right]$, it would come at the cost of a substantially increased number of parameters. Section 4.4 will provide an evaluation of the empirical importance of cross-equation correlation among parameters, showing that the model is not restrictive in practice. An additional note of prudence is necessary: in fact, if a full correlation structure among parameters exists, it would probably show up in a dynamic setting, especially if learning plays a role in the economy. This potential kind of correlation is absent in the model.

\section{A Small Model of the US Economy}

The techniques just described are applied for the estimation of a small quarterly model of the US economy. Three variables are included in the model: inflation rate and unemployment rate, representing the non-policy block and a short-term nominal interest rate, representing the policy block. ${ }^{9}$ Even though most of the literature has preferred larger sets of variables (see, for instance, Bernanke and Mihov, 1998, Christiano, Eichenbaum and Evans, 1999, Leeper, Sims and Zha, 1996), there are a few existing papers that use the same small system of equations (for example Rotemberg and Woodford, 1997, Cogley and Sargent, 2001 and 2003, Stock and Watson, 2001). Here, the choice is mostly due to the attempt to reduce the number of parameters of the model. As already mentioned, the alternative would be to use a wider set of variables at the cost of tighter priors, necessary to avoid ill behaviors. ${ }^{10}$

The sample runs from 1953:I to 2001:III. Two lags are used for the estimation. The simulations

\footnotetext{
${ }^{9}$ All series are taken from the Standard and Poor's DRI database. Inflation is measured by the annual growth rate of a chain weighted GDP price index. Unemployment is the unemployment rate referred to all workers over 16. The nominal interest rate is the yield on 3-month Treasury bills, preferred to the more conventional federal fund rate because available for a longer period of time.

10 In order to solve this problem, an interesting line for future research would be to assume the existence of common factors driving the dynamics of the coefficients. This approach would be very useful to reduce the rank of the variance covariance matrix of the innovations to the coefficients on the lags. The idea is the following: replace (5) with $B_{t}=B_{t-1}+C \xi_{t}$, where $\xi_{t}$ has dimension lower than $B_{t}$. This can be handled by the estimation procedure of section 3, adding an extra step in order to draw the $C$ matrix. The state equation can be rewritten as $B_{t}=B_{0}+C \sum_{s=1}^{t} \xi_{s}$ and substituted in the measurement equation (4). Conditional on the rest of the parameters, the posterior of vec(C) turns out to be normally distributed with known mean and variance.
} 
are based on 10,000 iterations of the Gibbs sampler, discarding the first 2,000 for convergence. As shown in appendix B, the sample autocorrelation functions of the draws decay quite fast and the convergence checks are satisfactory.

\subsection{Priors}

The first ten years (forty observations, from 1953:I to 1962:IV) are used to calibrate the prior distributions. For example, the mean and the variance of $B_{0}$ are chosen to be the $O L S$ point estimates $\left(\widehat{B}_{O L S}\right)$ and four times its variance in a time invariant VAR, estimated on the small initial subsample. In the same way, a reasonable prior for $A_{0}$ can be obtained. For $\log \sigma_{0}$ instead, the mean of the distribution is chosen to be the logarithm of the $O L S$ point estimates of the standard errors of the same time invariant VAR, while the variance covariance matrix is arbitrarily assumed to be the identity matrix. Finally, degrees of freedom and scale matrices are needed for the inverse-Wishart prior distributions of the hyperparameters. The degrees of freedom are set to 4 for $W$ and 2 and 3 for the two blocks of $S$ (basically, one plus the dimension of each matrix). The reason why the degrees of freedom are chosen differently is that for the inverse-Wishart distribution to be proper the degrees of freedom must exceed the dimension respectively of $W$ and the blocks of $S$. For $Q$ the degrees of freedom are set to 40 (the size of the previous initial subsample), since a slightly tighter prior seems to be necessary in order to avoid implausible behaviors of the time varying coefficients (see section 4.4 for a detailed discussion). Following the literature (Cogley, 2003, Cogley and Sargent 2001 and 2003), the scale matrices, $\bar{Q}, \bar{W}, \bar{S}_{1}$ and $\bar{S}_{2}$, are chosen to be constant fractions of the variances of the corresponding $O L S$ estimates on the initial subsample (multiplied by the degrees of freedom, because, in the inverse-Wishart distribution, the scale matrix has the interpretation of sum of squared residuals). Summarizing, the priors take the form:

$$
\begin{aligned}
B_{0} & \sim N\left(\widehat{B}_{O L S}, 4 \cdot V\left(\widehat{B}_{O L S}\right)\right), \\
A_{0} & \sim N\left(\widehat{A}_{O L S}, 4 \cdot V\left(\widehat{A}_{O L S}\right)\right), \\
\log \sigma_{0} & \sim N\left(\log \widehat{\sigma}_{O L S}, I_{n}\right), \\
Q & \sim I W\left(k_{Q}^{2} \cdot 40 \cdot V\left(\widehat{B}_{O L S}\right), 40\right), \\
W & \sim I W\left(k_{W}^{2} \cdot 4 \cdot I_{n}, 4\right) \\
S_{1} & \sim I W\left(k_{S}^{2} \cdot 2 \cdot V\left(\widehat{A}_{1, O L S}\right), 2\right), \\
S_{2} & \sim I W\left(k_{S}^{2} \cdot 3 \cdot V\left(\widehat{A}_{2, O L S}\right), 3\right),
\end{aligned}
$$

where $S_{1}$ and $S_{2}$ denote the two blocks of $S$, while $\widehat{A}_{1, O L S}$ and $\widehat{A}_{2, O L S}$ stand for the two correspondent blocks of $\widehat{A}_{O L S}$. The benchmark results presented in this section are obtained using the following values: $k_{Q}=0.01, k_{S}=0.1, k_{W}=0.01$. Set in this way, the priors are not flat, but diffuse and uninformative. The reader is referred to section 4.4 for a detailed discussion of this choice and of the robustness of the results to alternative prior specifications. The discussion of the empirical results starts with the analysis of time variation of the US non-systematic monetary policy. 


\subsection{Non-systematic monetary policy}

The term non-systematic monetary policy is used to capture both "policy mistakes" and interest rate movements that are responses to variables other than inflation and unemployment (therefore exogenous in this setup). Identified monetary policy shocks are the measure of non-systematic policy actions. The identifying assumption for the monetary policy shocks is that monetary policy actions affect inflation and unemployment with at least one period of lag. Therefore, interest rates are ordered last in the VAR. It is important to stress that this is not an ordering issue (like the one highlighted in section 3.1), but an identification condition, essential to isolate monetary policy shocks. Moreover, this identification assumption is completely standard in most of the existing literature (for instance Leeper, Sims and Zha, 1996, Rotemberg and Woodford, 1997, Bernanke and Mihov, 1998, Christiano, Eichenbaum and Evans, 1999). On the other hand, the simultaneous interaction between inflation and unemployment is arbitrarily modeled in a lower triangular form, with inflation first. As opposed to the previous one, this is not an identification condition, but a necessary normalization. While this arbitrary normalization could potentially make a difference (for the reasons illustrated in section 3.1), in the context of this empirical application, it turns out that the ordering of the non-policy block does not affect the results.

If identified monetary policy shocks are the measure of non-systematic policy actions, it seems natural to measure the relative importance and changes of non-systematic monetary policy by the time varying standard deviation of the identified monetary policy shocks. Figure 1c presents a plot of the posterior mean and the 16 th and 84 th percentiles $^{11}$ of the time varying standard deviation of the monetary policy shocks. This graph presents at least two interesting features. The period 7983 exhibits a substantially higher variance of monetary policy shocks. This is not surprising at all and confirms that the Volcker regime and his monetary aggregates targeting was actually peculiar in the history of US monetary policy. What is more interesting is the fact that the volatility of monetary policy shocks was on average higher in the pre-Volcker period than in the post-Volcker one, when it is very low and substantially constant. This suggests that Taylor-type rules (like the one estimated in this paper) are very good approximations of the US monetary policy in the last fifteen years, while it is likely that in the 60 s and 70 s the Fed was responding to other variables than just inflation and unemployment. ${ }^{12}$ The result that monetary policy shocks were more volatile before 1983 is robust to other specifications of the interest rate equation. One obvious alternative is to use the interest rates in logs instead of levels and, consequently, interpret the coefficients of the interest rate equation as semi-elasticities. This modification of the baseline setting allows the interest rate responses to inflation and real activity to differ, depending on the level of the interest rate itself. In other words, high responses (and, therefore, large interest rate variability) are more likely when interest rates are high and viceversa. This specification of the policy equation has been

\footnotetext{
${ }^{11}$ Under normality, the 16 th and 84 th percentiles correspond to the bounds of a one-standard deviation confidence interval.

${ }^{12}$ Interestingly, figure 1 also shows how, not only monetary policy shocks, but also the shocks to the inflation and unemployment equations have a lower standard deviation in the second part of the sample.
} 
also suggested by Sims (1999 and 2001a). The estimation of the model using the log of the interest rate does not change the main characteristics of figure 1c. Overall, the result that the volatility of monetary policy shocks, is higher in the pre-Volcker period and in the first half of the Volcker chairmanship is robust and consistent with Bernanke and Mihov (1998), Sims (1999 and 2001a) and Sims and Zha (2004).

The changes in the effects of non-systematic policy are summarized in figures 2 and 3. Figures $2 \mathrm{a}$ and $3 \mathrm{a}$ plot the impulse responses of inflation and unemployment to a monetary policy shock in three different dates of the sample. The other graphs of figures 2 and 3 represent pairwise differences between impulse responses in different dates with the 16th and 84th percentiles. The dates chosen for the comparison are 1975:I, 1981:III and 1996:I. They are somehow representative of the typical economic conditions of the chairmanships of Burns, Volcker and Greenspan, but, apart from that, they are chosen arbitrarily. ${ }^{13}$ Clearly, these responses do not vary much over time, indicating that the estimated coefficients do not show much time variation. Some differences are detectable in the responses of inflation which, in the Burns and Volcker periods, exhibit a small price puzzle. The price puzzle almost disappears in the Greenspan period. However such effect does not seem to be statistically significant once standard errors are taken into account. Moreover, the stability of the response of unemployment to policy shocks is remarkable.

Summarizing, there is no evidence of nonlinearities in the responses of the economy to nonsystematic policy.

\subsection{Systematic monetary policy}

Common and theoretically important measures of the degree of activism of the systematic monetary policy are the responses of the interest rate to inflation and unemployment. To be concrete, consider the modified version of the monetary policy rule of Clarida, Galí and Gertler (2000), given by the following equations:

$$
\begin{aligned}
i_{t} & =\rho(L) i_{t-1}+(1-\rho(1)) i_{t}^{*}+e_{t}^{m p} \\
i_{t}^{*} & =i^{*}+\tilde{\phi}(L)\left(\pi_{t}-\pi^{*}\right) .
\end{aligned}
$$

$i_{t}$ is a short-term interest rate; $i_{t}^{*}$ is a target for the short-term interest rate; $\pi_{t}$ is the inflation rate; $\pi^{*}$ is the target for inflation; $i^{*}$ is the desired interest rate when inflation is at its target; $e^{m p}$ is a monetary policy shock; $\rho(L)$ and $\tilde{\phi}(L)$ are lag polynomials. In other words, the assumption is that the monetary authorities specify a target for the short-term interest rate, but they achieve it gradually (because they tend to smooth interest rate movements). Finally, observe that, just for simplicity, (12) does not allow for any policy response to real activity. The combination of (11) and

\footnotetext{
${ }^{13}$ Actually 1975:I is a NBER business cycle trough date, 1981:III is a NBER business cycle peak date and 1996:I is the middle date between the last NBER trough and peak dates (1991:I and 2001:I). They are meant to capture very different economic conditions. Experiments with different dates and with averages over the periods 1970:I 1978:I, 1979:IV - 1983:IV and 1987:III - 2001:III (Burns chairmanship, Volcker monetary targeting and Greenspan chairmanship) give very similar conclusions.
} 
(12) gives

$$
i_{t}=\bar{i}+\rho(L) i_{t-1}+\phi(L) \pi_{t}+e_{t}^{m p},
$$

where $\bar{i} \equiv(1-\rho(1))\left(i^{*}-\tilde{\phi}(1) \pi^{*}\right)$ and $\phi(L) \equiv(1-\rho(1)) \tilde{\phi}(L)$. (13) is interpretable as a Taylor rule, augmented in order to consider dynamically richer interest rate responses to inflation. In the context of the popular New-Keynesian framework (see Woodford, 2003 for an overview), it has been argued that the Taylor principle is a necessary and sufficient condition under which the rational expectation equilibrium exhibits desirable properties, as determinacy and expectational stability (Woodford, 2001 and 2003, Bullard and Mitra, 2000). ${ }^{14}$ In this general framework, the Taylor principle requires that

$$
\tilde{\phi}(1)>1
$$

i.e. that the sum of coefficients of the target interest rate response to inflation must be bigger than one. Observe that, in this framework, $\tilde{\phi}(1)$ coincides with (and, consequently, provides an alternative interpretation for) $\frac{\phi(1)}{(1-\rho(1))}$, i.e. the long run interest rate response to inflation, implied by the policy rule. Therefore, from a theoretical point of view, long run responses are fundamental. From an empirical point of view, finite time responses of the interest rate to unemployment and inflation seem more informative and realistic quantities. Recall that the time varying variance covariance matrix plays a key role in the analysis of the simultaneous and long run responses that follows.

Figures 4 and 6 present a plot of the evolution of the responses of interest rate to a one percent permanent increase in inflation and unemployment over the last forty years. Chart (a) represents the simultaneous response, charts (b),(c) and (d) the responses after 10, 20 and 60 quarters. 16th and 84th percentiles are also reported. Even if not exactly the same, the 60 quarters response may be thought as a long run response (or, alternatively, the response of the target interest rate) and therefore can be used to check the Taylor principle. ${ }^{15}$

A few results stand out. First, the correlation between simultaneous and long run responses to inflation is high (figure 5), even though for the simultaneous response the upward sloping trend is less pronounced. Second, the response of interest rate to inflation is often gradual. In other words, it takes time for the interest rate to reach the long run response level after an inflationary shock. Notice that usually, less aggressive contemporaneous responses reach approximately the same level of the aggressive responses in less than ten quarters. The dynamic response to unemployment behaves quite differently. As shown in figure 7, the contemporaneous response is almost the same as the long run one, suggesting that the Fed reacts to unemployment much faster than to inflation.

\footnotetext{
${ }^{14}$ The Taylor principle is only sufficient for determinacy and expectational stability if the monetary policy rule include an interest rate response to real activity (Woodford, 2001).

15 Note that the uncertainty around these responses increases with time. In particular, the posterior is clearly skewed toward high level for the response to inflation and low levels for the response to unemployment. This is due to the persistence of the interest rate that implies a sum of coefficients often close to one. This makes the long run responses explode. For the same reason, the graphs report posterior medians instead of posterior means (which would be even higher in absolute value). Finally, notice that the responses do not include the uncertainty about the future evolution of the coefficients.
} 
This is not surprising and due to the fact that the signal-to-noise ratio for unemployment is probably perceived to be higher than for inflation. Apart from that, responses to inflation and unemployment are quite highly correlated. This indicates that the periods in which the Fed is more aggressive against inflation are the same periods in which monetary policy is more reactive to unemployment fluctuations.

Third, looking at long run reactions, as many authors have emphasized, systematic monetary policy has become more reactive to inflation and unemployment over the last twenty years with respect to the $60 \mathrm{~s}$ and $70 \mathrm{~s}$. However, the $60 \mathrm{~s}$ and $70 \mathrm{~s}$ do not seem to be characterized by a violation of the Taylor principle. In fact, the long run reaction of the interest rate to inflation remains above one for the whole sample period. This result differs from large part of the literature that has found an interest rate reaction to inflation lower than one before the beginning of the 80s (among others see Judd and Rudebusch, 1998, Clarida, Galí and Gertler, 2000 and Cogley and Sargent, 2001). This difference can be explained by the fact that this paper presents smoothed estimates that use all the information of the sample, as opposed to filtered estimates that use only the information contained in the relevant subsample. Strictly related to this, another reason that may drive the conclusions reached by the previous literature is the fact that subsample estimates may suffer from the well known bias toward stationarity that characterizes many dynamic models estimated on small samples. If for some reasons (as it seems to be) the bias toward stationarity is larger in the subsample of the $60 \mathrm{~s}$ and 70 s this creates a larger bias of the long run reactions of interest rate toward low levels. ${ }^{16}$

Fourth, neither the simultaneous reactions nor the long run ones seem to exhibit an absorbing state, but rather a frequent alternation among states. This result partly confirms Sims (1999 and 2001a) view of non-unidirectional regime switches, even though the upward sloping trend of the long run response to inflation provides some evidence of a learning pattern toward a more aggressive policy.

In order to establish the responsibility of the Fed in the high inflation and unemployment episodes of the $70 \mathrm{~s}$ and early $80 \mathrm{~s}$, it is particularly interesting to analyze the effects of these changes of the systematic part of the policy on the rest of the economy. The methodology to do so is straightforward: observing the data, all the parameters of the model can be drawn from the joint posterior (as described in section 3 and appendix A). For every draw it is possible to reconstruct the i.i.d. sequence of unit-variance structural shocks of the model. Starting from 1970:I these shocks can be used to simulate counterfactual data, constructed using different values of the parameters. These new series can be interpreted as the realization of the data that would have been observed had the parameters of the models been the ones used to generate the series. In the context of this paper, the interesting experiment is "planting Greenspan into the 70s". In other words, the idea consists of replaying history drawing the parameters of the policy rule in the 70s from their

\footnotetext{
${ }^{16}$ A way of checking the presence of a strong bias is to estimate a time invariant VAR on the relevant sample and construct a 95 percent confidence interval based on the implied unconditional distribution. If the initial conditions of the variables lie way outside the interval, this is a signal of the presence of some sort of bias. See Sims (2000) for details.
} 
posterior in 1991-1992, ${ }^{17}$ in order to see if this would have made any difference. This is done in figure 8 , from which it is clear that the counterfactual paths of inflation and unemployment do not differ much from the actual ones. Notice that a very similar conclusion is reached if history is replayed using in the 70s the standard deviation of the monetary policy shocks drawn from its 1991-1992 posterior.

Of course, a Lucas (1976) critique issue arises in this type of counterfactual analysis. If the policy rules adopted in the 70s and in the 90 s were different, rational and forward looking private agents would take this into account and modify their behavior accordingly. Therefore, the interpretation of "Greenspan into the 70s" is not straightforward, since the counterfactual exercise takes the private sector behavior in the 70s as given and unchanged. However, there are many reasons why doing the exercise is interesting anyway. First, the effects of the Lucas critique are drastically mitigated in any Bayesian framework, in which policy is random. This argument is even stronger in this framework, in which there is an explicit model of the stochastic time variation of policy. Second, according to the previous estimates, the changes in policy between the Burns period and the Greenspan period do not seem to be drastic enough to generate a different, previously unknown regime. In other words, the policy parameters of 1991-1992 seem to belong to the private sector probability distribution over the policy parameters for almost all the 1970-1987 period, as it appears from figures 4 and 6 (even though these figures show the smoothed estimates of the posteriors instead of the filtered ones). Third, the Lucas critique would be a major problem if the counterfactual paths of figure 8 were very different from the actual paths of inflation and unemployment. Since they are not, it is less clear why rational private agents would modify their behavior in response to changes of the policy rule, which have minor implications. Fourth, even when the counterfactual experiment is repeated, drawing not only the monetary policy, but also the private sector's parameters from their 1991-1992 posterior, the picture (not reported) looks fundamentally unchanged.

Overall, these findings are in contrast with the anecdotal view that monetary policy was very loose in the 70s and this caused the poor economic performance of that decade. On the contrary, the impression is that the differences in the conduct of systematic interest rate policies under Burns, Volcker and Greenspan were not large enough to have any relevant effect on the dynamics of inflation and unemployment. Indeed, additional counterfactual simulations (not reported) show that the peaks of inflation and unemployment of the 70s and early 80 s seem to be better (although not completely) explained by the high volatility of the shocks that characterized those periods. Figures $1 \mathrm{a}$ and $1 \mathrm{~b}$ show clearly that inflation and unemployment residuals were less volatile after 1985. Inflation residuals' variability reaches its peak around 1975, oscillating and finally declining since 1982. Less smooth seem to be the changes in the unemployment residuals' variability. In particular, the convergence toward a more stable regime starts with a rapid volatility decrease between 1983 and 1985. This findings are in line with other studies as Blanchard and Simon

\footnotetext{
17 1991-1992 posterior means the posterior of the average value of the parameters in the eight quarters of 1991 and 1992. Again, the choice of the dates is arbitrary, meant to capture a period of relative economic stability, with relatively strong reactions to unemployment and inflation.
} 
(2001), Hanson (2003), Sims and Zha (2004), Stock and Watson (2002). ${ }^{18}$

Of course, the small model used in this paper is not able to address the issue of the true behavioral source of these sequences of particularly bad shocks. However, it is able to assert that this source is neither a monetary policy sector specified as a Taylor rule, nor a stylized private sector block with only inflation and real activity.

\subsection{Sensitivity to priors and robustness to alternative specifications}

This subsection shows robustness of the empirical results to alternative priors and specifications.

a. Priors and model's fit The main results of section 4 were presented for the particular prior specification chosen in section 4.1. This subsection justifies this choice and demonstrates the robustness of the empirical conclusions to alternative prior specifications. While the choice of the priors for the initial states is completely innocuous, ${ }^{19}$ the selection of $k_{Q}, k_{S}$ and $k_{W}$ turns out to be more important. Especially for $k_{Q}$ this is not surprising: in a VAR with three variables and two lags the $Q$ matrix contains 231 free parameters. With such a high number of free parameters, the specification of a sensible prior becomes essential, in order to prevent cases of ill-determinations like the ones described in section 3.

While posterior inference can in principle be affected by the choice of $k_{Q}, k_{S}$ and $k_{W}$, it is worth noting that $k_{Q}, k_{S}$ and $k_{W}$ do not parameterize time variation, but just prior beliefs about the amount of time variation. In large samples, as usual, the posterior mean converges to the maximum likelihood estimator. Consider as an example the matrix $Q$, which represents the amount of time variation in the coefficients ( $B$ 's). The random variable $Q \mid B^{T}$ is distributed as an inverse-Wishart. It can be shown that the conditional posterior mean has the following form:

$$
E\left(Q \mid B^{T}\right)=\frac{v}{v+T} \frac{\bar{Q}}{v}+\frac{T}{v+T} Q^{*}
$$

where $\bar{Q}$ is the prior scale matrix, $v$ are the prior degrees of freedom, $Q^{*}$ is the maximum likelihood estimate of $Q$ (conditional on $B^{T}$ ) and $T$ is the sample size. ${ }^{20}$ In this framework, the choice of $k_{Q}$ would affect $\bar{Q}$. Given (14), it is easy to see that $k_{Q}$ parameterizes time variation only when the prior degrees of freedom are driven to infinity. In general the posterior mean will be a combination of the prior and likelihood information, where the weights are determined by the relative size of the degrees of freedom of the prior and the sample size.

\footnotetext{
${ }^{18}$ It is worth pointing out that the data reject also the hypothesis that changes in the conduct of monetary policy have influenced inflation and unemployment through an indirect channel, i.e. by affecting the variability of nonpolicy shocks. In fact, the correlation between the innovations to the reactions to inflation and unemployment and the innovations to the variances of inflation and unemployment are estimated to be essentially equal to zero (once the estimation algorithm is extended to take into account the possibility that these correlations are different from zero).

19 Much flatter specifications of these priors (for example with variances ten or twenty times bigger) deliver exactly the same results.

${ }^{20}$ Remember that the scale matrix in the inverse-Wishart parameterization has the interpretation of sum of squared residuals. Therefore $\frac{\bar{Q}}{v}$ is interpretable as a variance and is comparable to $Q^{*}$.
} 
This being said, three reasons lead to the choice of $k_{Q}=0.01$ in the benchmark model. First, the model seems to misbehave if a higher $k_{Q}$ is chosen. It can be shown that, in the benchmark case of $k_{Q}=0.01$, the prior mean for $Q$ implies a $95 \%$ probability of a $78 \%$ average cumulative change of the coefficients over the relevant sample. This is saying that $k_{Q}=0.01$ is a value that does not particularly penalize time variation in the coefficients. With a prior that explicitly favors a higher degree of time variation, the coefficients change considerably over time, but just in order to explain outliers and to push the in-sample error to zero. Their time variation captures much more high frequency variation than the one that would be captured by a pure random walk. In other words, most of the variation of the data seems to be explained by the raw shocks as opposed to the dynamics of the model. ${ }^{21}$ A strange behavior such as this is typical of very narrow likelihood peaks in very uninteresting regions of the parameter space, where the level of the likelihood is not informative of the model's fit. Moreover, any selection criterion based formally (Uhlig, 2001) or informally on the shape of the impulse responses would reject this model, which exhibits responses often exploding and with counterintuitive signs.

The second reason for choosing $k_{Q}=0.01$ is being consistent with the literature. Cogley and Sargent (2001) use the same value. Stock and Watson (1996) experiment with values of $k_{Q}$ higher and lower than 0.01, pointing out that models with large a priori time variation do poorly in forecasting. Notice that using a lower $k_{Q}$ would only reinforce the conclusion that changes in the conduct of monetary policy are not responsible for the economic instability of the 70s.

The third reason that justifies the choice of $k_{Q}=0.01$ is a formal model selection. Proper posterior probabilities for a set of models (characterized by different prior assumptions on the time variation of the unobservable states) are computed. These are the probabilities of each model, once all the other parameters are integrated out. They are computed according to the reversible jump Markov chain Monte Carlo (RJMCMC) method described in Dellaportas, Forster and Ntzoufras (2002), that extends the original contribution of Carlin and Chib (1995). In the context of static and dynamic factor models, Lopes and West (2000) and Justiniano (2004) have shown that RJMCMC outperforms alternative methods, based on the computation of the marginal likelihood (for an overview, see Justiniano, 2004). The details of the application to this model are provided in appendix C. The posterior probabilities are computed for 18 models, represented by all possible combinations of $k_{Q}=\{0.01 ; 0.05 ; 0.1\}, k_{S}=\{0.01 ; 0.1 ; 1\}$ and $k_{W}=\{0.001 ; 0.01\}$. Doing model selection to choose among models with different priors might seem unusual. However, notice that this procedure is equivalent to imposing a single, more complex prior, given by a mixture of the original, simple priors. Independently of the initial value of the chain, the model characterized by $k_{Q}=0.01, k_{S}=0.1$ and $k_{W}=0.01$ gets a posterior probability that is essentially one. The fact that only one of the models gets positive posterior probability is not surprising and just indicates that the models' space is sparse. Observe that, while in some applications this is undesirable, here

${ }^{21}$ The same type of misbehavior appears if the prior degrees of freedom for $Q$ are set to 22 (which is the minimum in order to have a proper prior), unless $k_{Q}$ is also reduced to approximately 0.003 . In this case the results are very similar to the ones of the benchmark case of section 4.2 and 4.3 . 
this is not a problem. In fact, the objective is just avoiding of imposing priors that are clearly at odds with the data. Finally, notice that the selected model is the one with the smallest value of $k_{Q}$. As argued above, this value of $k_{Q}$ is not very restrictive against time variation in the coefficients of the lag structure. Yet, the results of section 4.2 and 4.3 seem to suggest that the amount of time variation of the coefficients of the lag structure is small relative to the time variation of the covariance matrix. Therefore, while in principle possible, it does not seem necessary to extend the model selection to include even smaller values of $k_{Q}$. A similar arguments applies to high values of $k_{W}$.

Given that the introduction of a time varying $A_{t}$ matrix is one of the contributions of this paper, there is no literature to refer to for a choice of $k_{S}$. Therefore, $k_{S}$ is fixed to 0.1 (the value of the best fitting model) for the benchmark results. Also the choice of $k_{W}=0.01$ is motivated by the results of the model selection procedure. In addition, the model is estimated also for other possible values of $k_{S}$ and $k_{W}$. The results (not reported) obtained with $k_{S}=1$ are very similar to the benchmark case. Instead, when $k_{S}$ is set to 0.01 , the changes in the interest rate responses to inflation and real activity are smaller and smoother. If anything, this reinforces the conclusion that changes in the conduct of monetary policy are not responsible for the economic instability of the 70 s. If $k_{W}$ is set to 0.001 the results are very much the same as in the baseline case. This supports the idea that time variation in the volatilities is fundamental to improve the fit of the model.

b. Random walk assumption As discussed in section 2, the random walk assumption for the evolution of the coefficients and log variances has some undesirable implications. In particular, it implies that the correlation among the reduced form residuals may become arbitrarily large in absolute value. To show that these undesirable implications are not to be taken too seriously, the model is re-estimated allowing the log standard errors and the coefficients of the simultaneous relations to follow more general AR processes. In a first experiment the autoregressive coefficients are all set to 0.95 (instead of 1 as in the random walk assumption), producing results not relevantly different from the ones presented above. In a second experiment these coefficients are estimated, by inserting an additional step in the Gibbs sampler. ${ }^{22}$ Again, the results produced by such a model are in line with the results of the benchmark model. The AR coefficients for the log standard errors of the residuals are estimated to be very high (between .95 and 1), while the AR coefficients for the simultaneous relations are estimated lower (between .5 and .9). The only consequence of this fact is that the model captures many temporary parameters shifts, other than the permanent ones.

c. Cross-equation restrictions The main results of section 4 are presented for the case of $S$ being block diagonal, with block corresponding to parameters belonging to separate equations. This choice simplifies the inference, but may be seen as an important restriction. To verify that

\footnotetext{
${ }^{22}$ Drawn the states with the usual Gibbs procedure for state-space forms, it is easy to draw the AR coefficients of the states evolution equation. The posterior is in fact normal, centered on the OLS coefficient with variance equal to the OLS variance.
} 
the results are not affected by such a choice, this assumption is now relaxed, allowing $S$ to be unrestricted. This involves a modification of the basic Markov chain Monte Carlo algorithm, as reported in appendix D. The model is re-estimated with this modified algorithm and the results are very similar to the main ones of section $4 .^{23}$ The only notable difference is that in the case of an unrestricted $S$ the estimates of the simultaneous relations exhibit slightly less time variation than in the benchmark case. Again, if anything, this strengthens the idea that changes in policy are not very important to explain the outbursts of inflation and unemployment. Furthermore, the correlations of the innovations to the coefficients of different equations are estimated to be basically zero.

In order to get some further insight on the relevance of cross-equation restrictions for the application to the US data, the model is re-estimated eliminating all the cross-equation restrictions, also in the lag structure. In practice, both $S$ and $Q$ are now assumed to be block diagonal, with the blocks corresponding to elements of the same equation. The results do not differ in any relevant way from the benchmark case, suggesting that cross-equation restrictions are not quantitatively very important.

\section{Conclusions}

This paper applies Markov chain Monte Carlo methods to the estimation of a time varying structural VAR. The sources of time variation are both the coefficients and, most importantly, the variance covariance matrix of the shocks. The time variation in the variance covariance matrix of the innovations not only seems to be empirically very important, but is also crucial to analyze the dynamics of the contemporaneous relations among the variables of the system.

In particular, the paper focuses on the role of monetary policy in the dynamics of inflation and unemployment for the US economy. There is evidence of time variation in the US monetary policy. Non-systematic policy has changed considerably over the last forty years, becoming less important in the last part of the sample. Systematic monetary policy on the other hand has become more aggressive against inflation and unemployment. Nevertheless, there seems to be little evidence for a causal link between changes in interest rates systematic responses and the high inflation and unemployment episodes. It is important to stress that this is not a statement about neutrality of monetary policy. For example, it is quite conceivable that monetary policy which does not respond to inflation at all, would introduce substantial instability in the economy. The estimates indicate, however, that within the range of policy parameters in the post-war era, the differences between regimes were not large enough to explain a substantial part of the fluctuations in inflation and unemployment. In particular, the less aggressive policy pursued by Burns in the 70s was

\footnotetext{
${ }^{23}$ The model is re-estimated using both the proposal distributions suggested in appendix D. In both cases the acceptance rate of the Metropolis-Hastings step is approximately 12 percent and the estimates are very similar to each other. The 12 percent acceptance rate seems acceptable, even though it is clear that the convergence is much slower than in the baseline case. This might also explain why the estimates of the simultaneous relations exhibit slightly less time variation than in the benchmark case.
} 
not loose enough to cause the great stagflation that followed. Indeed, the peaks in inflation and unemployment of the 70s and early 80 s seem to be better explained by non-policy shocks than weaker interest rate responses to inflation and real activity.

In order to explore the true behavioral sources of these sequences of particularly bad shocks, a larger and fully structural model is needed. This suggests a clear direction for future research, namely allowing for a general form of simultaneity, overidentifying assumptions, cross-equation restrictions in the VAR representation and for a larger set of variables, solving the problem of the high number of parameters. A natural approach seems to be assuming the existence of common factors driving the dynamics of the coefficients. 


\section{A The basic Markov chain Monte Carlo algorithm}

\section{A.1 Step 1: Drawing coefficient states}

Conditional on $A^{T}, \Sigma^{T}$ and $V$, the observation equation (4) is linear and has Gaussian innovations with known variance. As shown in Fruhwirth-Schnatter (1994) and Carter and Kohn (1994), the density $p\left(B^{T} \mid y^{T}, A^{T}, \Sigma^{T}, V\right)$ can be factored as

$$
p\left(B^{T} \mid y^{T}, A^{T}, \Sigma^{T}, V\right)=p\left(B_{T} \mid y^{T}, A^{T}, \Sigma^{T}, V\right) \prod_{t=1}^{T-1} p\left(B_{t} \mid B_{t+1}, y^{t}, A^{T}, \Sigma^{T}, V\right),
$$

where

$$
\begin{aligned}
B_{t} & \mid B_{t+1}, y^{t}, A^{T}, \Sigma^{T}, V \sim N\left(B_{t \mid t+1}, P_{t \mid t+1}\right), \\
B_{t \mid t+1} & =E\left(B_{t} \mid B_{t+1}, y^{t}, A^{T}, \Sigma^{T}, V\right), \\
P_{t \mid t+1} & =\operatorname{Var}\left(B_{t} \mid B_{t+1}, y^{t}, A^{T}, \Sigma^{T}, V\right) .
\end{aligned}
$$

$p(\cdot)$ is used to denote a generic density function, while $N$ denotes the Gaussian distribution. The vector of $B$ 's can be easily drawn because $B_{t \mid t+1}$ and $P_{t \mid t+1}$ can be computed using the forward (Kalman filter) and the backward recursions reported in appendix A.6, applied to the state space form given by (4) and (5). Specifically, the last recursion of the filter provides $B_{T \mid T}$ and $P_{T \mid T}$, i.e. mean and variance of the posterior distribution of $B_{T}$. Drawn a value from this distribution, the draw is used in the backward recursion to obtain $B_{T-1 \mid T}$ and $P_{T-1 \mid T}$ and so on. ${ }^{24}$

\section{A.2 Step 2: Drawing covariance states}

The system of equations (4) can be written as

$$
A_{t}\left(y_{t}-X_{t}^{\prime} B_{t}\right)=A_{t} \widehat{y}_{t}=\Sigma_{t} \varepsilon_{t},
$$

where, taking $B^{T}$ as given, $\widehat{y}_{t}$ is observable. Since $A_{t}$ is a lower triangular matrix with ones on the main diagonal, (15) can be rewritten as

$$
\widehat{y}_{t}=Z_{t} \alpha_{t}+\Sigma_{t} \varepsilon_{t}
$$

$\alpha_{t}$ is defined in (6) and $Z_{t}$ is the following $n \times \frac{n(n-1)}{2}$ matrix:

$$
Z_{t}=\left[\begin{array}{cccc}
0 & \cdots & \cdots & 0 \\
-\widehat{y}_{1, t} & 0 & \cdots & 0 \\
0 & -\widehat{y}_{[1,2], t} & \ddots & \vdots \\
\vdots & \ddots & \ddots & 0 \\
0 & \cdots & 0 & -\widehat{y}_{[1, \ldots, n-1], t}
\end{array}\right]
$$

\footnotetext{
${ }^{24}$ For further details on Gibbs sampling for state space models see Carter and Kohn (1994).
} 
where, abusing notation, $\widehat{y}_{[1, \ldots, i], t}$ denotes the row vector $\left[\widehat{y}_{1, t}, \widehat{y}_{2, t}, \ldots, \widehat{y}_{i, t}\right]$.

Observe that the model given by (16) and (6) has a Gaussian but nonlinear state space representation. The problem, intuitively, is that the dependent variable of the observation equation, $\widehat{y}_{t}$, appears also on the right hand side in $Z_{t}$. Therefore, the vector $\left[\widehat{y}_{t}, \widehat{\alpha}_{t}\right]$ is not jointly normal and thus, the conditional distributions cannot be computed using the standard Kalman filter recursion. However, under the additional assumption of $S$ block diagonal, this problem can be solved by applying the Kalman filter and the backward recursion equation by equation. This is not only because the dependent variable of each equation, $\widehat{y}_{i, t}$, does not show up on the right hand side of the same equation, but also because the vector $\widehat{y}_{[1, \ldots, i-1], t}$ can be treated as predetermined in the $i$-th equation, due to the triangular structure. Another way to see it is to reinterpret time as going through equations, that is treating different measurement equations as belonging to distinct and subsequent time periods. Exactly like in the previous step of the sampler, this procedure allows to recursively recover

$$
\begin{aligned}
\alpha_{i, t \mid t+1} & =E\left(\alpha_{i, t} \mid \alpha_{i, t+1}, y^{t}, B^{T}, \Sigma^{T}, V\right), \\
\Lambda_{i, t \mid t+1} & =\operatorname{Var}\left(\alpha_{i, t} \mid \alpha_{i, t+1}, y^{t}, B^{T}, \Sigma^{T}, V\right),
\end{aligned}
$$

where $\alpha_{i, t}$ is the $i$-th block of $\alpha_{t}$, corresponding to the coefficients of the $i$-th equation in (16). As above, $\alpha_{i, t}$ can be drawn recursively from $p\left(\alpha_{i, t} \mid \alpha_{i, t+1}, y^{t}, B^{T}, \Sigma^{T}, V\right)$, which is $N\left(\alpha_{i, t \mid t+1}, \Lambda_{i, t \mid t+1}\right)$.

Notice that without block diagonality of $S$ it would not be possible to apply the recursions equation by equation and to draw separately every block $\alpha_{i}^{T}$. However, while the $S$ block diagonal assumption simplifies the analysis, it is not essential and appendix D provides a modification of this algorithm in order to deal with an unrestricted $S$.

\section{A.3 Step 3: Drawing volatility states}

Consider now the system of equations

$$
A_{t}\left(y_{t}-X_{t}^{\prime} B_{t}\right)=y_{t}^{*}=\Sigma_{t} \varepsilon_{t}
$$

where, taking $B^{T}$ and $A^{T}$ as given, $y_{t}^{*}$ is observable. This is a system of nonlinear measurement equations, but can be easily converted in a linear one, by squaring and taking logarithms of every element of (17). Due to the fact that $y_{i, t}^{2}$ can be very small, an offset constant is used to make the estimation procedure more robust. This leads to the following approximating state space form:

$$
\begin{aligned}
y_{t}^{* *} & =2 h_{t}+e_{t} \\
h_{t} & =h_{t-1}+\eta_{t} .
\end{aligned}
$$

$y_{i, t}^{* *}=\log \left[\left(y_{i, t}^{*}\right)^{2}+\bar{c}\right] ; \bar{c}$ is the offset constant (set to 0.001); $e_{i, t}=\log \left(\varepsilon_{i, t}^{2}\right) ; h_{i, t}=\log \sigma_{i, t}$. Observe that the $e$ 's and the $\eta$ 's are not correlated. ${ }^{25}$

\footnotetext{
${ }^{25}$ This is easy to see since the $\varepsilon$ 's and the $\eta$ 's are independent. However, even if the $\varepsilon$ 's and the $\eta$ 's were not independent, the $e$ 's and the $\eta$ 's would be uncorrelated. For a proof of this result, see Harvey, Ruiz and Shephard (1994).
} 
The system in this form has a linear, but non-Gaussian state space form, because the innovations in the measurement equations are distributed as a $\log \chi^{2}(1)$. In order to further transform the system in a Gaussian one, a mixture of normals approximation of the $\log \chi^{2}$ distribution is used, as described in Kim, Shephard and Chib (1998). Observe that the variance covariance matrix of the $\varepsilon$ 's is the identity matrix. This implies that the variance covariance matrix of the $e$ 's is also diagonal, allowing to use the same (independent) mixture of normals approximation for any element of $e$. Kim, Shephard and Chib (1998) select a mixture of 7 normal densities with component probabilities $q_{j}$, means $m_{j}-1.2704$, and variances $v_{j}^{2}, j=1, \ldots, 7$. The constants $\left\{q_{j}, m_{j}, v_{j}^{2}\right\}$ are chosen to match a number of moments of the $\log \chi^{2}(1)$ distribution. Appendix A.7 reports the constants $\left\{q_{j}, m_{j}, v_{j}^{2}\right\} .^{26}$

Define $s^{T}=\left[s_{1}, \ldots, s_{T}\right]^{\prime}$, the matrix of indicator variables selecting at every point in time which member of the mixture of normal approximation has to be used for each element of $e$. Conditional on $B^{T}, A^{T}, V$ and $s^{T}$, the system has an approximate linear and Gaussian state space form. Again, exactly like in the previous steps of the sampler, this procedure allows to recursively recover

$$
\begin{aligned}
h_{t \mid t+1} & =E\left(h_{t} \mid h_{t+1}, y^{t}, A^{T}, B^{T}, V, s^{T}\right), \\
H_{t \mid t+1} & =\operatorname{Var}\left(h_{t} \mid h_{t+1}, y^{t}, A^{T}, B^{T}, V, s^{T}\right)
\end{aligned}
$$

and recursively draw every $h_{t}$ from $p\left(h_{t} \mid h_{t+1}, y^{t}, A^{T}, B^{T}, V, s^{T}\right)$, which is $N\left(h_{t \mid t+1}, H_{t \mid t+1}\right) .{ }^{27}$

Conditional on $y^{* * T}$ and the new $h^{T}$, it is possible to sample the new $s^{T}$ matrix, to be used in the next iteration. As in Kim, Shephard and Chib (1998), this is easily done by independently sampling each $s_{i, t}$ from the discrete density defined by

$$
\operatorname{Pr}\left(s_{i, t}=j \mid y_{i, t}^{* *}, h_{i, t}\right) \propto q_{j} f_{N}\left(y_{i, t}^{* *} \mid 2 h_{i, t}+m_{j}-1.2704, v_{j}^{2}\right), \quad j=1, \ldots, 7, \quad i=1, \ldots, n
$$

\section{A.4 Step 4: Drawing hyperparameters}

The hyperparameters of the model are the diagonal blocks of $V: Q, W$ and the diagonal blocks of $S$ (corresponding to parameters belonging to different equations). Conditional on $B^{T}, \Sigma^{T}, A^{T}$ and $y^{T}$, each square block has an inverse-Wishart posterior distribution, independent of the other blocks. Moreover, conditional on $B^{T}, \Sigma^{T}, A^{T}$ and $y^{T}$, it is easy to draw from these inverse-Wishart posteriors because the innovations are observable. ${ }^{28}$

\footnotetext{
${ }^{26}$ In this paper the reweighting procedure used in Kim, Shephard and Chib (1998) to correct the minor approximation error is not adopted.

27 Cogley (2003) and Cogley and Sargent (2003) have a similar model for the variances. However, to evaluate the posterior they adopt the algorithm suggested by Jacquier, Polson and Rossi (1994), that draws the volatility states one at a time. As pointed out in Carter and Kohn (1994) and Kim, Shephard and Chib (1998), this increases drastically the autocorrelations of the draws and decreases efficiency. Uhlig (1997) has a similar model with constant coefficients. However he adopts nonlinear filtering procedures and importance sampling to evaluate the posterior. As stressed by Uhlig himself, importance sampling procedure are subject to the risk of real inefficient sampling, in the cases in which, out of thousands draws, only a few of them have a non-zero weight.

${ }^{28}$ See Gelman et al. (1995) for a description of the sampling procedure from a normal-inverse-Wishart distribution, also when combined with natural conjugate priors.
} 


\section{A.5 Summary}

To summarize, the sampler takes the form:

a. Initialize $A^{T}, \Sigma^{T}, s^{T}$ and $V$.

b. Sample $B^{T}$ from $p\left(B^{T} \mid y^{T}, A^{T}, \Sigma^{T}, V\right)$.

c. Sample $A^{T}$ from $p\left(A^{T} \mid y^{T}, B^{T}, \Sigma^{T}, V\right)$.

d. Sample $\Sigma^{T}$ from $p\left(\Sigma^{T} \mid y^{T}, A^{T}, B^{T}, s^{T}, V\right)$.

e. Sample $s^{T}$ from $p\left(s^{T} \mid y^{T}, A^{T}, \Sigma^{T}, V\right)$.

f. Sample $V$, by sampling $Q, W$ and $S$ from $p\left(Q, W, S \mid y^{T}, B^{T}, A^{T}, \Sigma^{T}\right)=p\left(Q \mid y^{T}, B^{T}, A^{T}, \Sigma^{T}\right)$. $p\left(W \mid y^{T}, B^{T}, A^{T}, \Sigma^{T}\right) \cdot p\left(S_{1} \mid y^{T}, B^{T}, A^{T}, \Sigma^{T}\right) \cdot \ldots \cdot p\left(S_{n-1} \mid y^{T}, B^{T}, A^{T}, \Sigma^{T}\right)$.

g. Go to b.

\section{A.6 Gibbs sampling for state space models}

Consider a measurement equation:

$$
y_{t}=H_{t} \beta_{t}+\varepsilon_{t}
$$

and a transition equation

$$
\beta_{t}=F \beta_{t-1}+u_{t}
$$

where

$$
\left[\begin{array}{l}
\varepsilon_{t} \\
u_{t}
\end{array}\right] \sim \text { i.i.d. } N\left(\left[\begin{array}{l}
0 \\
0
\end{array}\right],\left[\begin{array}{cc}
R_{t} & 0 \\
0 & Q
\end{array}\right]\right) .
$$

Let

$$
\begin{aligned}
\beta_{t \mid s} & =E\left(\beta_{t} \mid Y^{s}, H^{s}, R^{s}, Q\right), \\
V_{t \mid s} & =\operatorname{Var}\left(\beta_{t} \mid Y^{s}, H^{s}, R^{s}, Q\right) .
\end{aligned}
$$

Then, given $\beta_{0 \mid 0}$ and $V_{0 \mid 0}$, a standard Kalman filter delivers:

$$
\begin{aligned}
\beta_{t \mid t-1} & =F \beta_{t-1 \mid t-1} \\
V_{t \mid t-1} & =F V_{t-1 \mid t-1} F^{\prime}+Q \\
K_{t} & =V_{t \mid t-1} H_{t}^{\prime}\left(H_{t} V_{t \mid t-1} H_{t}^{\prime}+R_{t}\right)^{-1} \\
\beta_{t \mid t} & =\beta_{t \mid t-1}+K_{t}\left(y_{t}-H_{t} \beta_{t \mid t-1}\right) \\
V_{t \mid t} & =V_{t \mid t-1}-K_{t} H_{t} V_{t \mid t-1}
\end{aligned}
$$


The last elements of the recursion are $\beta_{T \mid T}$ and $V_{T \mid T}$, which are the mean and the variance of the normal distribution used to make a draw for $\beta_{T}$. The draw of $\beta_{T}$ and the output of the filter are now used for the first step of the backward recursion, which provides $\beta_{T-1 \mid T}$ and $V_{T-1 \mid T}$, used to to make a draw of $\beta_{T-1}$. The backward recursion continues until time zero. For a generic time $t$, the updating formulas of the backward recursion are:

$$
\begin{aligned}
\beta_{t \mid t+1} & =\beta_{t \mid t}+V_{t \mid t} F^{\prime} V_{t+1 \mid t}^{-1}\left(\beta_{t+1}-F \beta_{t \mid t}\right), \\
V_{t \mid t+1} & =V_{t \mid t}-V_{t \mid t} F^{\prime} V_{t+1 \mid t}^{-1} F V_{t \mid t} .
\end{aligned}
$$

\section{A.7 Selection of the mixing distribution to be $\log \chi^{2}(1)$ :}

\begin{tabular}{cccc}
\hline \hline$\omega$ & $q_{j}=\operatorname{Pr}(\omega=j)$ & $m_{j}$ & $v_{j}^{2}$ \\
\hline \hline 1 & 0.00730 & -10.12999 & 5.79596 \\
2 & 0.10556 & -3.97281 & 2.61369 \\
3 & 0.00002 & -8.56686 & 5.17950 \\
4 & 0.04395 & 2.77786 & 0.16735 \\
5 & 0.34001 & 0.61942 & 0.64009 \\
6 & 0.24566 & 1.79518 & 0.34023 \\
7 & 0.25750 & -1.08819 & 1.26261 \\
\hline \hline \multicolumn{4}{c}{ Source: Kim, Shephard and Chib (1998). }
\end{tabular}

\section{B Convergence of the Markov chain Monte Carlo algorithm}

This appendix assesses convergence of the Markov chain Monte Carlo algorithm in the baseline application to the US data. ${ }^{29}$

First of all, different starting points of the chain (randomly selected) produce the same results. These results are also not sensitive to the initial number of discarded draws or the total number of passes of the Gibbs sampler (an experiment with 30,000 iterations, keeping one every three draws, gave the same results). For space reasons, a complete description of the characteristics of the chain is provided only for 706 parameters of the model: the hyperparameters (the 241 free elements of $V)$ and the volatilities (3 sets of volatility states for 155 time periods).

In order to judge how well the chain mixes, common practice is to look at the autocorrelation function of the draws. Low autocorrelations suggest that the draws are almost independent, which increases the efficiency of the algorithm. Figure 9a plots the 20th order sample autocorrelation of the draws. In all three panels of figure 9, points $1-241$ correspond to the free elements of $V$ (first the variances and then the covariances by row), while points $242-706$ correspond to the volatilities (for every time period, by equation). With some exceptions the plot remains below 0.2

\footnotetext{
${ }^{29}$ For the convergence checks, I used modified versions of codes of Alejandro Justiniano and James P. LeSage.
} 
and often below 0.1. Closely related, figure $9 \mathrm{~b}$ presents the inefficiency factors (IFs) for the posterior estimates of the parameters. The IF is the inverse of the relative numerical efficiency measure of Geweke's (1992), i.e. the IF is an estimate of $\left(1+2 \sum_{k=1}^{\infty} \rho_{k}\right)$, where $\rho_{k}$ is the $k$-th autocorrelation of the chain. In this application the estimate is performed using a 4 percent tapered window for the estimation of the spectral density at frequency zero. Values of the IFs below or around twenty are regarded as satisfactory. Again, except for a few cases, the IFs are around or even below 20. The table below summarizes the distribution of the IFs for the posterior estimates of all different sets of parameters. On average, the set of parameters with the highest IFs are the $A$ 's, for which it is not uncommon to have IFs between 4 and 75 . In all other cases, $90 \%$ of the IFs are below 25 . The IFs are remarkably low for the time varying coefficients ( $B$ 's).

\begin{tabular}{c|c|c|c|c|c|c}
\hline \hline & median & mean & min & max & 10th percentile & 90th percentile \\
\hline \hline$V$ & 18.99 & 22.29 & 9.63 & 185.51 & 12.76 & 25.60 \\
\hline$\Sigma$ & 7.49 & 9.61 & 1.62 & 45.61 & 4.31 & 18.80 \\
\hline$A$ & 20.01 & 30.32 & 2.07 & 118.67 & 4.85 & 75.81 \\
\hline$B$ & 5.63 & 6.86 & 2.34 & 18.50 & 2.94 & 13.61 \\
\hline \hline
\end{tabular}

Summary of the distribution of the Inefficiency Factors for different sets of parameters. $V$ : elements of the covariance matrix of the model's innovations; $\Sigma$ : time varying volatilities; $A$ : time varying simultaneous relations; $B$ : time varying coefficients.

As a further check, figure 9c plots the Raftery and Lewis' (1992) diagnostic of the total number of runs required to achieve a certain precision (the parameters for the diagnostic are specified as follows: quantile $=0.025$; desired accuracy $=0.025$; required probability of attaining the required accuracy $=0.95)$. The required number of runs is always much below the total number of iterations of this application. To conclude, considering the very high dimensionality of the problem, the convergence diagnostics seem satisfactory.

\section{Reversible jump Markov chain Monte Carlo (RJMCMC)}

This appendix describes the RJMCMC algorithm used for the model selection. Further details on the method are in Dellaportas, Forster and Ntzoufras (2002), Lopes and West (2000) and Justiniano (2004).

Consider a set $M$ of competing models. In the case of this paper different models are characterized by different prior assumptions on the time variation of the unobservable states, as described in section 4.1. For every model $m \in M$, define the set of model's parameters

$$
\theta^{m}=\left\{\alpha_{1}^{m}, \ldots, \alpha_{T}^{m}, \log \sigma_{1}^{m}, \ldots, \log \sigma_{T}^{m}, Q^{m}, S^{m}, W^{m}\right\} .
$$


In very general terms, the method consists of simulating the joint posterior probability distribution of the couple $\left(m, \theta^{m}\right)$. This can be done using a standard Metropolis-Hastings approach. A proposal value $\left(m^{\prime}, \theta^{m^{\prime}}\right)$ is generated from a proposal distribution $q_{p}\left(m^{\prime}, \theta^{m^{\prime}}\right)=q\left(\theta^{m^{\prime}} \mid m^{\prime}\right) \cdot J\left(m^{\prime}\right)$. The new value is accepted with the usual Metropolis-Hastings acceptance probability (stated below). If the proposal value is rejected, it is replaced by the previous element of the chain. This procedure generates a set of values, which can be used to approximate the posterior of $\left(m, \theta^{m}\right)$ and, among other things, to compute posterior probabilities over the models' space.

The details of the algorithm adopted in the paper are as follows:

a. For every model $m \in M$, the output of a preliminary run of the Gibbs sampler is used to construct the following, independent proposal distributions for the elements of $\theta^{m}$ :

$$
\begin{aligned}
q\left(\alpha_{t}^{m} \mid m\right)= & N\left(\overline{\alpha_{t}^{m}}, 2 \cdot \overline{\operatorname{var}\left(\alpha_{t}^{m}\right)}\right), \\
q\left(\log \sigma_{t}^{m} \mid m\right)= & N\left(\overline{\log \sigma_{t}^{m}}, 2 \cdot \overline{\operatorname{var}\left(\log \sigma_{t}^{m}\right)}\right), \\
q\left(Q^{m} \mid m\right)= & I W\left(40 \cdot \overline{Q^{m}}, 40\right), \\
q\left(W^{m} \mid m\right)= & I W\left(4 \cdot \overline{W^{m}}, 4\right), \\
q\left(S_{1}^{m} \mid m\right)= & I W\left(2 \cdot \overline{S_{1}^{m}}, 2\right), \\
& \vdots \\
q\left(S_{n-1}^{m} \mid m\right)= & I W\left((n-1) \cdot \overline{S_{n-1}^{m}}, n-1\right),
\end{aligned}
$$

The variables denoted by the upper bar are posterior means of the preliminary Gibbs sampler, except for $\overline{\operatorname{var}\left(\alpha_{t}^{m}\right)}$ and $\overline{\operatorname{var}\left(\log \sigma_{t}^{m}\right)}$ that are posterior variances. In practice, the preliminary Gibbs run is used to center approximate posteriors, that will be adopted as proposal distributions. The approximate posteriors are made more diffuse than the exact ones on purpose, in order to facilitate the convergence of the algorithm. In this paper the preliminary Gibbs samplers are based on 2,000 iterations, discarding the first 500 .

b. Initialize $m$ and draw a value of $\theta^{m}$ from the proposal distribution $q\left(\theta^{m} \mid m\right)$.

c. Draw a candidate couple $\left(m^{\prime}, \theta^{m^{\prime}}\right)$ from $q_{p}\left(m^{\prime}, \theta^{m^{\prime}}\right)$. This can be easily done by first drawing $m^{\prime}$ from a proposal distribution $J\left(m^{\prime}\right)$ over the models' space and then $\theta^{m^{\prime}}$ from $q\left(\theta^{m^{\prime}} \mid m^{\prime}\right)$.

d. Accept the new couple $\left(m^{\prime}, \theta^{m^{\prime}}\right)$ with probability

$$
a=\min \left\{\frac{p\left(y^{T} \mid m^{\prime}, \theta^{m^{\prime}}\right) p\left(\theta^{m^{\prime}} \mid m^{\prime}\right) p\left(m^{\prime}\right)}{p\left(y^{T} \mid m, \theta^{m}\right) p\left(\theta^{m} \mid m\right) p(m)} \frac{q\left(\theta^{m} \mid m\right) J(m)}{q\left(\theta^{m^{\prime}} \mid m^{\prime}\right) J\left(m^{\prime}\right)}, 1\right\},
$$

where $p\left(y^{T} \mid m, \theta^{m}\right)$ is the likelihood, computed through the Kalman filter to integrate out the $B$ 's; $p\left(\theta^{m} \mid m\right)$ is the prior of $\theta^{m}$ within model $m ; p(m)$ is the prior probability of model $m ; q\left(\theta^{m} \mid m\right)$ 
is the proposal distribution for the parameters, conditional on the model, constructed as above; $J(m)$ is the unconditional proposal distribution over the models. If the new draw is not accepted, keep the previous couple $\left(m, \theta^{m}\right)$.

e. Go to c.

\section{The Markov chain Monte Carlo algorithm in the case of $S$ un- restricted}

This appendix presents an extension of the basic MCMC algorithm, in order to deal with the case of $S$ (the variance covariance matrix of the innovations to the simultaneous relations) left unrestricted. The modification works as follows: step 2 of the Gibbs sampler is replaced by a Metropolis-Hastings step in which a candidate value for the $\alpha^{\prime} \mathrm{s}, \alpha^{T *}$, is drawn from a proposal distribution $f_{P}\left(\alpha^{T} \mid y^{T}, B^{T}, \Sigma^{T}, V\right)$. The candidate value is accepted with probability equal to

$$
\min \left\{1, \frac{p\left(\alpha^{T *} \mid y^{T}, B^{T}, \Sigma^{T}, V\right) f_{P}\left(\alpha^{T,-1} \mid y^{T}, B^{T}, \Sigma^{T}, V\right)}{p\left(\alpha^{T,-1} \mid y^{T}, B^{T}, \Sigma^{T}, V\right) f_{P}\left(\alpha^{T *} \mid y^{T}, B^{T}, \Sigma^{T}, V\right)}\right\},
$$

where $p(\cdot)$ is the posterior of $\alpha^{T}$ and $\alpha^{T,-1}$ represents the previous draw of $\alpha^{T}$ in the chain. If the

candidate is rejected, $\alpha^{T}$ is set to be $\alpha^{T,-1}$. Step 4 is modified straightforwardly in order to sample $S$ jointly and not in blocks. As in the Gibbs sampling case, this procedure allows to draw from the exact joint posterior of the parameters. The last issue is related to the choice of the proposal distribution. A natural candidate is the distribution used to sample $\alpha^{T}$ under the assumption of $S$ block diagonal. Another possibility is to treat (erroneously) the state space form given by (16) and (6) as linear and draw a candidate value for $\alpha^{T}$ using the usual forward and backward recursions. Clearly, if the proposal distribution is not close to the true density convergence can be much slower than in the baseline case. In the context of the empirical application of this paper, with both the proposal distributions suggested above, the acceptance rate was approximately 12 percent. 


\section{References}

BERNANKE B. S. and I. MIHOV (1998), "Measuring Monetary Policy", The Quarterly Journal of Economics, 113, 869-902.

BLANCHARD O. and J. SIMON (2001), "The Long and Large Decline in U.S. Output Volatility", Brookings Papers on Economic Activity 1, 135-164.

BOIVIN J. (2001), “The Fed's Conduct of Monetary Policy: Has It Changed and Does It Matter?", Columbia Business School, mimeo.

BOIVIN J. and M. GIANNONI (2003), "Has Monetary Policy Become More Effective?", NBER working paper no. 9459 .

BULLARD J. and K. MITRA (2000), "Determinacy, Learnability, and Monetary Policy Inertia", working paper no. 200-030A, Federal Reserve Bank of St. Luis.

CARTER C. K. and R. KOHN (1994), "On Gibbs Sampling for State Space Models", Biometrika, $81,541-553$.

CARLIN B. P. and S. CHIB (1995), "Bayesian Model Choice via Markov Chain Monte Carlo Methods", Journal of Royal Statistical Society B, 157, 473-484.

CANOVA F. (1993), "Modelling and Forecasting Exchange Rates with a Bayesian Time-Varying Coefficient Model", Journal of Economic Dynamics and Control, 17, 233-261.

CHIB S., F. NARDARI and N. SHEPHARD (2002), "Analysis of High Dimensional Multivariate Stochastic Volatility Models", Nuffield College, University of Oxford, mimeo.

CHRISTIANO L. J., M. EICHENBAUM and C. EVANS (1999), "Monetary Shocks: What Have We Learned and To What End?", in J. B. Taylor and M. Woodford (Eds.), Handbook of Macroeconomics, Elsevier Science, North-Holland, New York, 1A, 65-148.

CICCARELLI M. and A. REBUCCI (2003), "Measuring Contagion with a Bayesian Time-Varying Coefficient Model", International Monetary Fund, mimeo.

COGLEY T. (2003), "How Fast Can the New Economy Grow? A Bayesian Analysis of the Evolution of Trend Growth", University of California, Davis, mimeo.

COGLEY T. and T.J. SARGENT (2001), "Evolving Post-World War II U.S. Inflation Dynamics", NBER Macroeconomics Annual, MIT Press, Cambridge, Massachusetts, 331-373.

COGLEY T. and T.J. SARGENT (2003), "Drifts and Volatilities: Monetary Policies and Outcomes in the Post WWII U.S.", New York University, mimeo. 
COX D. R. and N. WERMUTH (1996), Multivariate Dependencies - Models, Analysis and Interpretation, Chapman and Hall, London.

DELLAPORTAS P., J. J. FORSTER and I. NTZOUFRAS (2002), "On Bayesian Model and Variable Selection Using MCMC", Statistics and Computing, 12, 27-36.

DEMPSTER A. (1972), "Covariance Selection”, Biometrics, 28, 157-175.

DOUCET A., N. DE FREITAS and N. GORDON (2001), Sequential Monte Carlo Methods in Practice, Springer-Verlag, New York.

FAVERO, C. A. and R. ROVELLI (2003), "Macroeconomic Stability and the Preferences of the Fed. A Formal Analysis, 1961-1998", Journal of Money, Credit and Banking, 35(4), 545-556.

FRUHWIRTH-SCHNATTER S. (1994), "Data Augmentation and Dynamic Linear Models", Journal of Time Series Analysis, 15, 183-202.

GELMAN A., J. B. CARLIN, H. S. STERN and D. B. RUBIN (1995), Bayesian Data Analysis, Chapman and Hall, London.

GEWEKE J. (1992), "Evaluating the Accuracy of Sampling-Based Approaches to the Calculation of Posterior Moments", in J. M. Bernardo, J. Berger, A. P. Dawid and A. F. M. Smith (Eds.), Bayesian Statistics, Oxford University Press, Oxford, 169-193.

HAMILTON J. H. (1989), "A New Approach to the Economic Analysis of Nonstationary Time Series and the Business Cycle", Econometrica, 57(2), 357-384.

HANSON M. (2003), "Varying Monetary Policy Regimes: a Vector Autoregressive Investigation", Wesleyan University, mimeo.

HARVEY A. C., E. RUIZ and N. SHEPHARD (1994), "Multivariate Stochastic Variance Models", Review of Economic Studies, 61, 247-264.

JACQUIER E., N. G. POLSON and P. E. ROSSI (1994), "Bayesian Analysis of Stochastic Volatility Models", Journal of Business and Economic Statistics, 12, 371-417.

JACQUIER E., N. G. POLSON and P. E. ROSSI (1995), "Models and Priors for Multivariate Stochastic Volatility", CIRANO working paper, 1995-18.

JUDD J. and G. D. RUDEBUSCH (1998), "Taylor's Rule and the Fed: 1970-1997", Federal Reserve Bank of San Francisco Economic Review, 3, 3-16.

JUSTINIANO A. (2004), "Estimation and Model Selection in Dynamic Factor Analysis", International Monetary Fund, mimeo. 
KIM C. and C. R. NELSON (1999), State-Space Models with Regime Switching, MIT Press, Cambridge, Massachusetts.

KIM S., N. SHEPHARD and S. CHIB (1998), "Stochastic Volatility: Likelihood Inference and Comparison with ARCH Models", Review of Economic Studies, 65, 361-393.

LEEPER E. and T. ZHA (2002), "Modest Policy Intervention", NBER working paper no. 9192.

LEEPER E., C. A. SIMS and T. ZHA (1996), "What Does Monetary Policy Do?", Brookings Papers on Economic Activity 2, 1-78.

LOPES H. F. and M. WEST (2000), "Model Uncertainty in Factor Analysis", Duke University, mimeo.

LUBIK T. A. and F. SCHORFHEIDE (2004), "Testing for Indeterminacy: An Application to U.S. Monetary Policy", American Economic Review, 94(1), 190-217.

LUCAS R. E. (1976), "Econometric Policy Evaluation: a Critique", in K. Brunner and A. Meltzer (Eds.) The Phillips Curve and Labor Markets, Canergie-Rochester Series on Public Policy, 1, $19-46$.

PINHEIRO J. and D. BATES (1996), "Unconstrained Parametrizations for Variance-Covariance Matrices", Statistics and Computing, 11, 289-296.

POURAHMADI M. (1999), "Joint Mean-Covariance Models with Applications to Longitudinal Data: Unconstrained Parameterizations", Biometrika, 86, 677-690.

POURAHMADI M. (2000), "Maximum Likelihood Estimation for Generalized Linear Models for Multivariate Normal Covariance Matrices", Biometrika, 87, 425-435.

RAFTERY A. E. and S. LEWIS (1992), "How Many Iterations in the Gibbs Sampler?" in J. M. Bernardo, J. Berger, A. P. Dawid and A. F. M. Smith (Eds.), Bayesian Statistics, Oxford University Press, Oxford, 763-773.

ROTEMBERG J. J. and M. WOODFORD (1997), “An Optimization-Based Econometric Framework for the Evaluation of Monetary Policy", NBER Macroeconomics Annual, MIT Press, Cambridge, Massachusetts, 297-346.

SARGAN J. D. and A. BHARGAVA (1983), "Maximum Likelihood Estimation of Regression Models with First Order Moving Average Errors when the Root Lies on the Unit Circle", Econometrica, 51, 799-820.

SHEPHARD N. (1994a), "Local Scale Models: State Space Alternatives to Integrated GARCH Processes", Journal of Econometrics, 60, 181-202.

SHEPHARD N. (1994b), "Partial Non-Gaussian State Space", Biometrika, 81, 115-131. 
SHEPHARD N. (1996), "Statistical Aspects of ARCH and Stochastic Volatility", in D. R. Cox, D. V. Hinkley and O. E. Barndorff-Nielsen (Eds.), Time Series Models in Econometrics, Finance and Other Fields, Chapman and Hall, London, 1-67.

SHEPHARD N. and A. C. HARVEY (1990), "On the Probability of Estimating a Deterministic Component in the Local Level Model”, Journal of Time Series Analysis, 11(4), 339-347.

SIMS C. A. (1993), "A 9 Variable Probabilistic Macroeconomic Forecasting Model", in J. H. Stock and M. W. Watson (Eds.), Business Cycles, Indicators and Forecasting, NBER studies in business cycles volume 28, 179-214.

SIMS C. A. (1999), "Drift and Breaks in Monetary Policy", Princeton University, mimeo.

SIMS C. A. (2000), "Using a Likelihood Perspective to Sharpen Econometric Discourse: Three Examples", Journal of Econometrics, 95(2), 443-462.

SIMS C. A. (2001a), "Stability and instability in US monetary policy behavior", Princeton University, mimeo.

SIMS C. A. (2001b), "Comment on Sargent and Cogley's: 'Evolving Post World War II U.S. Inflation Dynamics"', NBER Macroeconomics Annual, MIT Press, Cambridge, Massachusetts, 373-379.

SIMS C. A. and T. ZHA (1998), "Bayesian Methods for Dynamics Multivariate Models", International Economic Review, 39, 949-968.

SIMS C. A. and T. ZHA (2004), "Were There Regime Switches in US Monetary Policy?", Princeton University, mimeo.

SMITH M. and R. KOHN (2002), "Parsimonious Covariance Matrix Estimation for Longitudinal Data", Journal of the American Statistical Association, 97, 1141-1153.

STOCK J. H. and M. W. WATSON (1996), "Evidence on Structural Instability in Macroeconomic Time Series Relations", Journal of Business and Economic Statistics, 14, 11-30.

STOCK J. H. and M. W. WATSON (1998), "Asymptotically Median Unbiased Estimation of Coefficient Variance in a Time Varying Parameter Model", Journal of the American Statistical Association, 93, 349-358.

STOCK J. H. and M. W. WATSON (2001), "Vector Autoregressions", Journal of Economic Perspectives, 15, 101-116.

STOCK J. H. and M. W. WATSON (2002), "Has the Business Cycle Changed and Why?", NBER working paper no. 9127. 
UHLIG H. (1997), "Bayesian Vector Autoregressions with Stochastic Volatility", Econometrica, $65,59-73$.

UHLIG H. (2001), "What Are the Effects of Monetary Policy on Output? Results from an Agnostic Identification Procedure", Tilburg University, mimeo.

WOODFORD M. (2001), "The Taylor Rule and Optimal Monetary Policy", American Economic Review, 91(2), 232-237.

WOODFORD M. (2003), Interest and Prices: Foundations of a Theory of Monetary Policy, Princeton University Press, Princeton, New Jersey. 
(a)

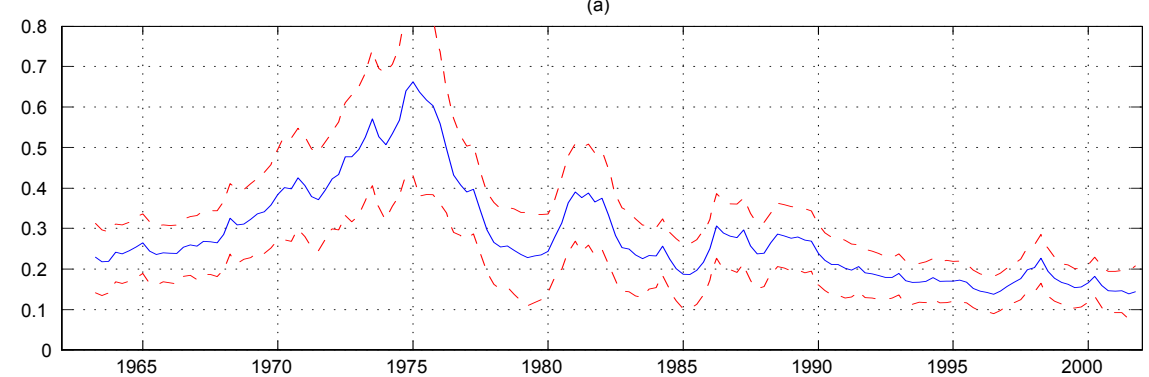

(b)

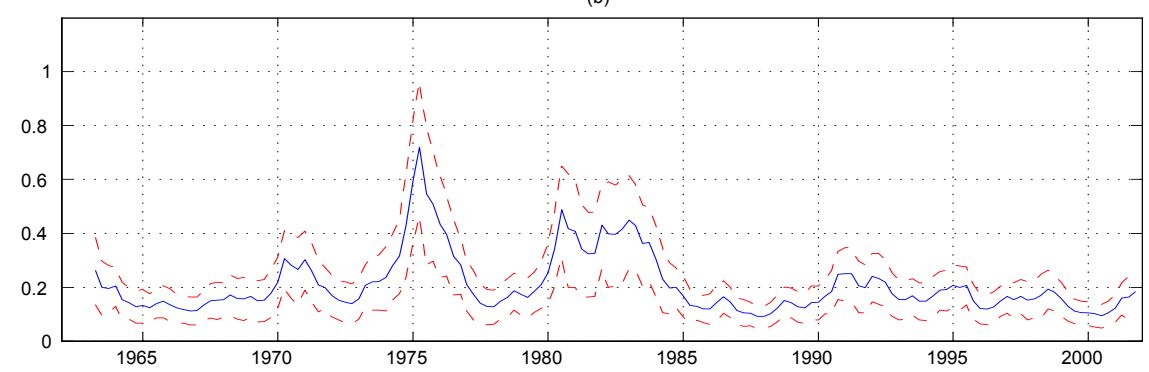

(c)

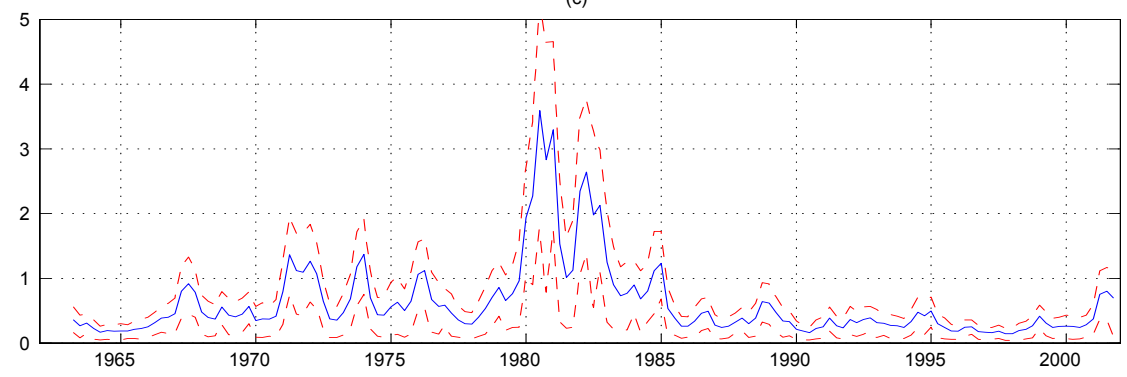

Figure 1: Posterior mean, 16th and 84th percentiles of the standard deviation of (a) residuals of the inflation equation, (b) residuals of the unemployment equation and (c) residuals of the interest rate equation or monetary policy shocks. 
(a)

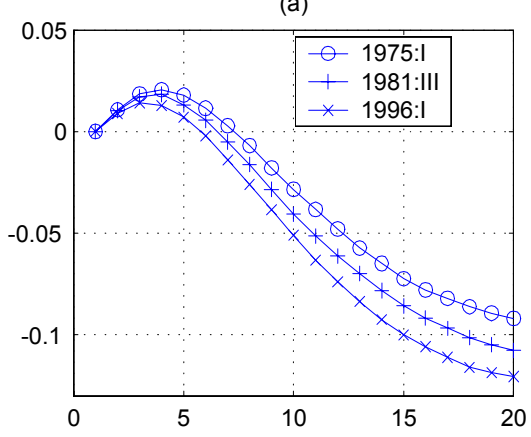

(c)

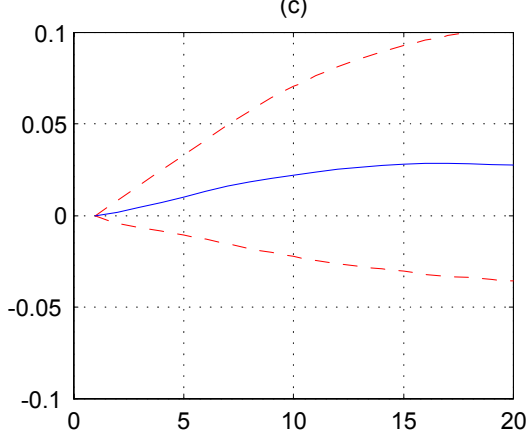

(b)

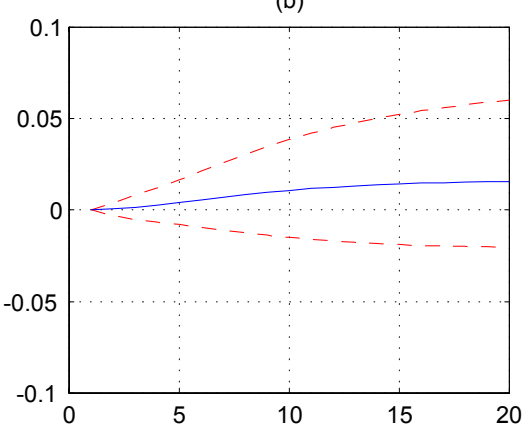

(d)

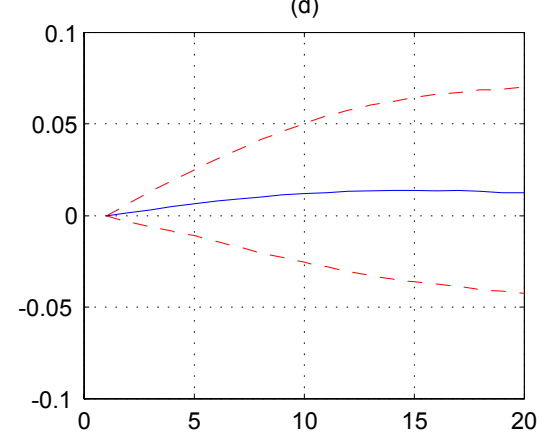

Figure 2: (a) impulse responses of inflation to monetary policy shocks in 1975:I, 1981:III and 1996:I, (b) difference between the responses in 1975:I and 1981:III with 16th and 84th percentiles, (c) difference between the responses in 1975:I and 1996:I with 16th and 84th percentiles, (d) difference between the responses in 1981:III and 1996:I with 16th and 84th percentiles. 
(a)

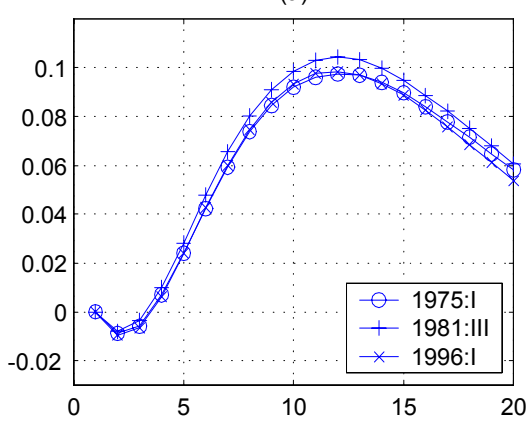

(c)

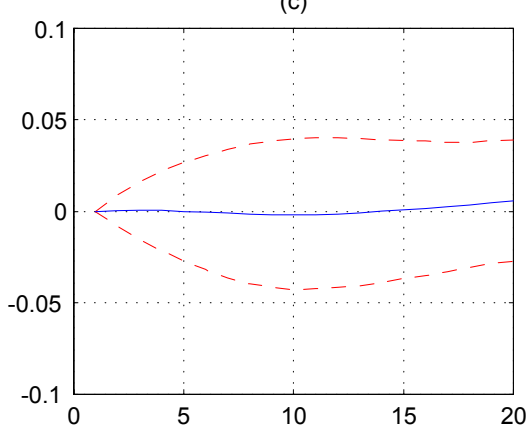

(b)

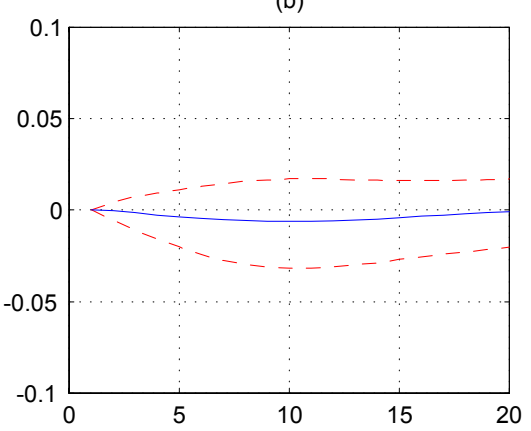

(d)

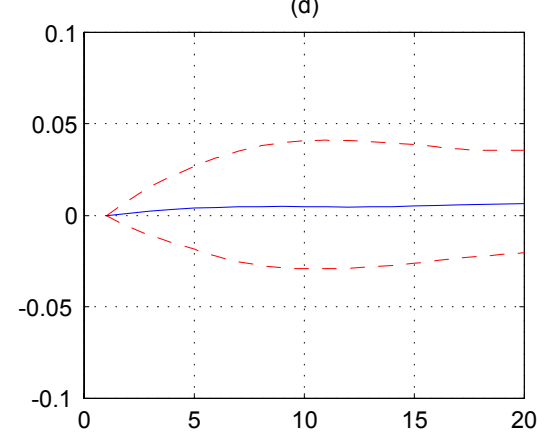

Figure 3: (a) impulse responses of unemployment to monetary policy shocks in 1975:I, 1981:III and 1996:I, (b) difference between the responses in 1975:I and 1981:III with 16th and 84th percentiles, (c) difference between the responses in 1975:I and 1996:I with 16th and 84th percentiles, (d) difference between the responses in 1981:III and 1996:I with 16th and 84th percentiles. 
(a)

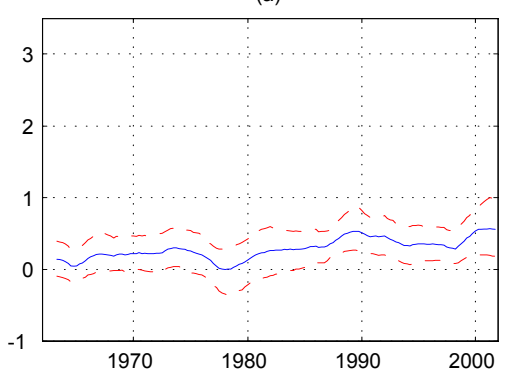

(c)

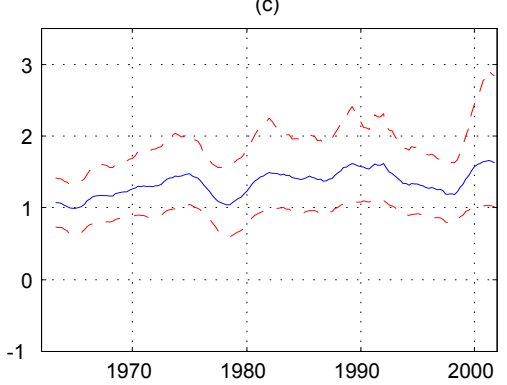

(b)

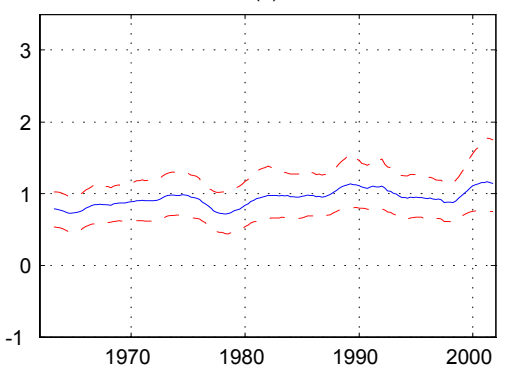

(d)

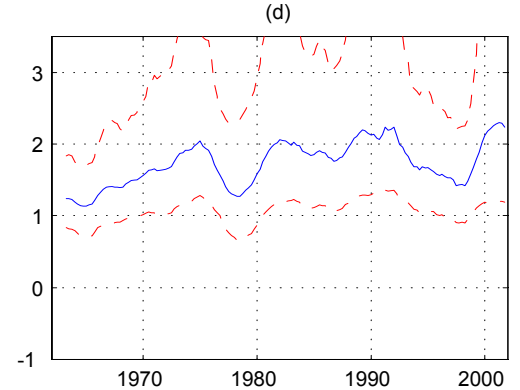

Figure 4: Interest rate response to a $1 \%$ permanent increase of inflation with 16 th and 84 th percentiles. (a) Simultaneous response, (b) response after 10 quarters, (c) response after 20 quarters, (d) response after 60 quarters.

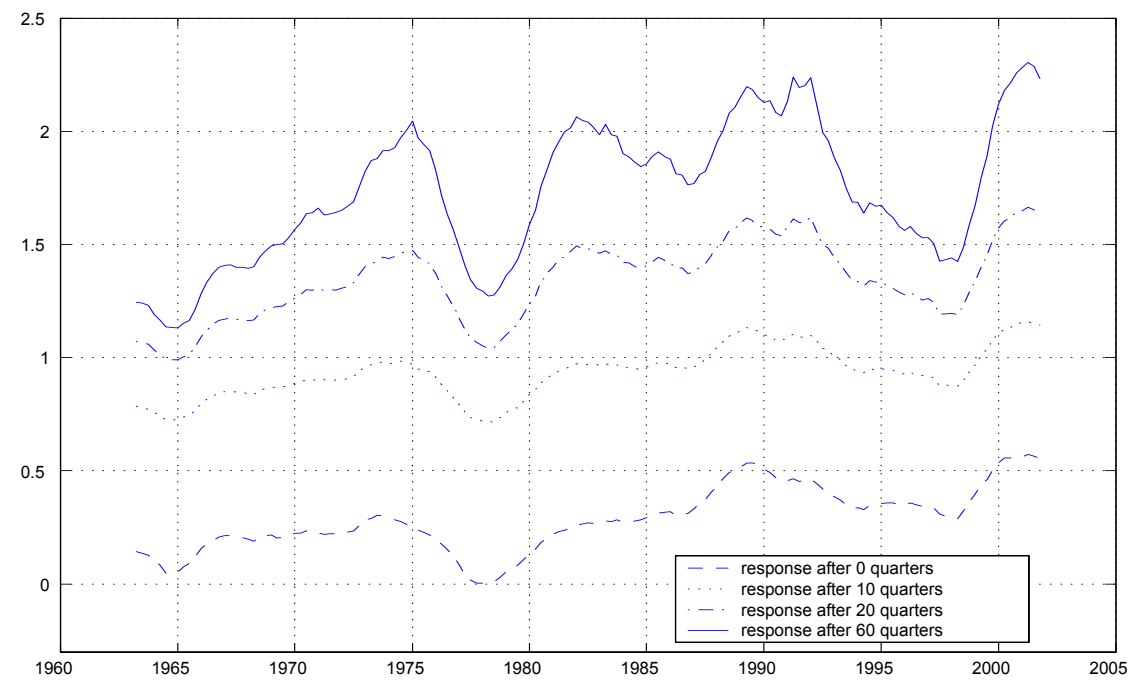

Figure 5: Interest rate response to a $1 \%$ permanent increase of inflation. 
(a)

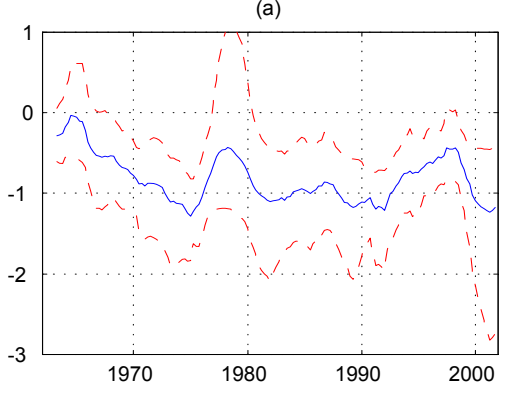

(c)

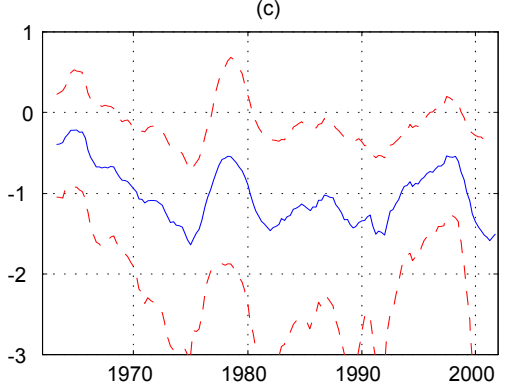

(b)

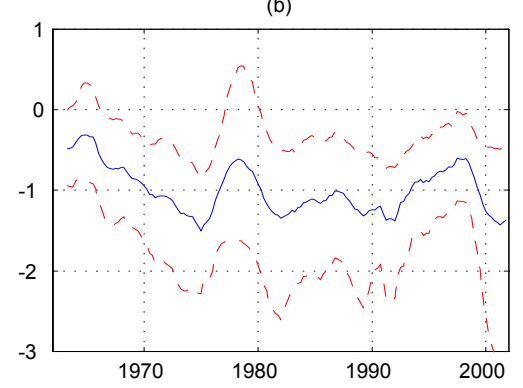

(d)

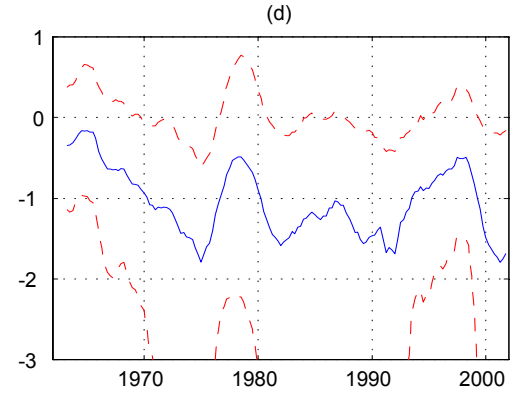

Figure 6: Interest rate response to a $1 \%$ permanent increase of unemployment with 16 th and 84th percentiles. (a) Simultaneous response, (b) response after 10 quarters, (c) response after 20 quarters, (d) response after 60 quarters.

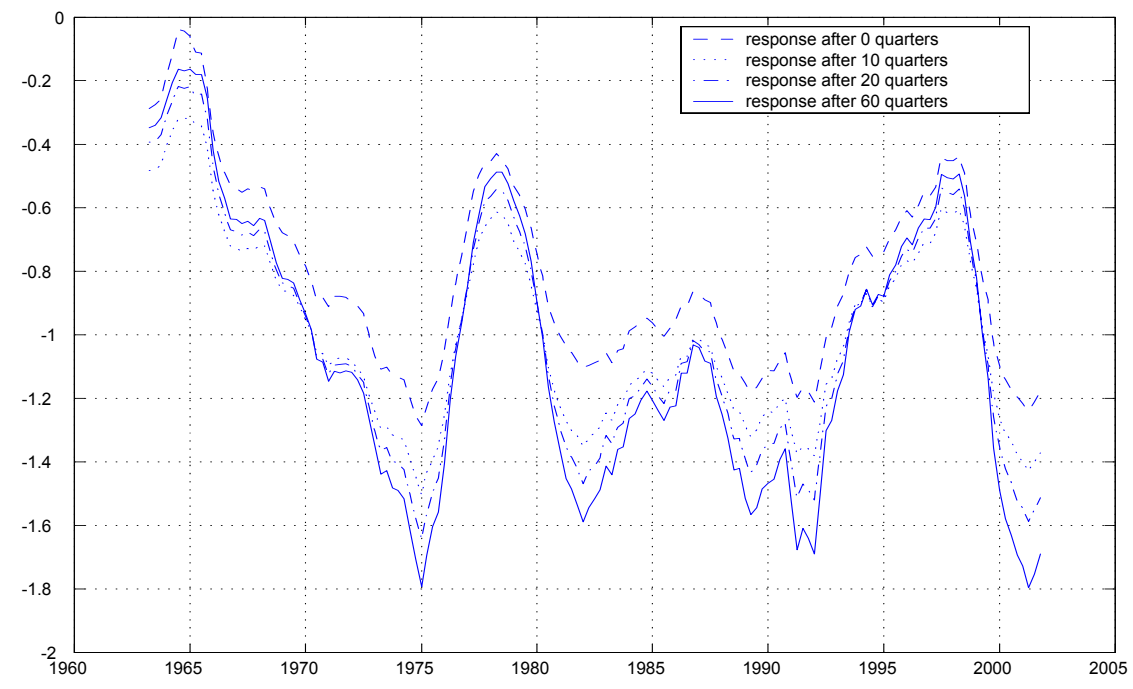

Figure 7: Interest rate response to a $1 \%$ permanent increase of unemployment. 

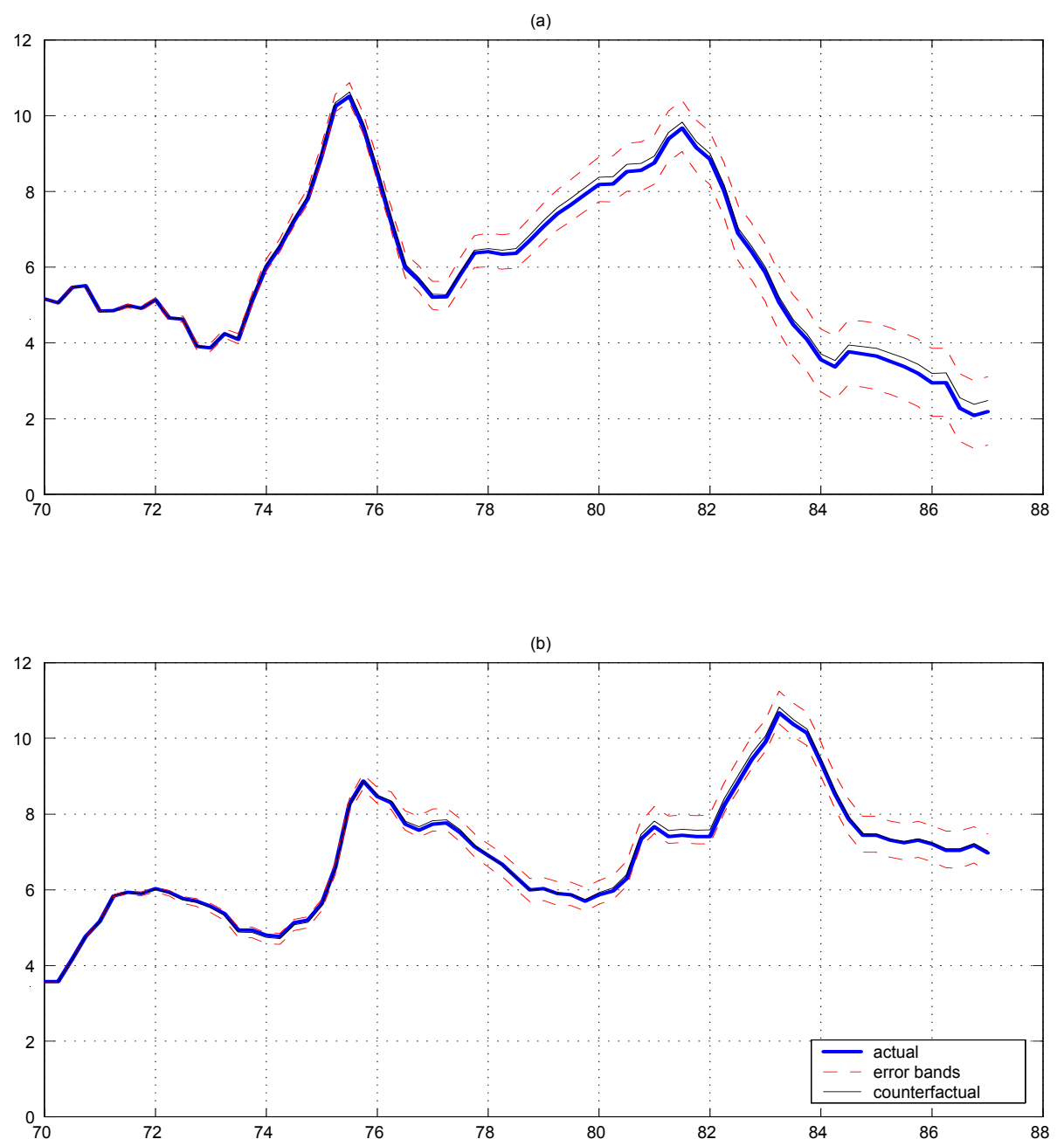

Figure 8: Counterfactual historical simulation drawing the parameters of the monetary policy rule from their 1991-1992 posterior. (a) Inflation, (b) unemployment. 


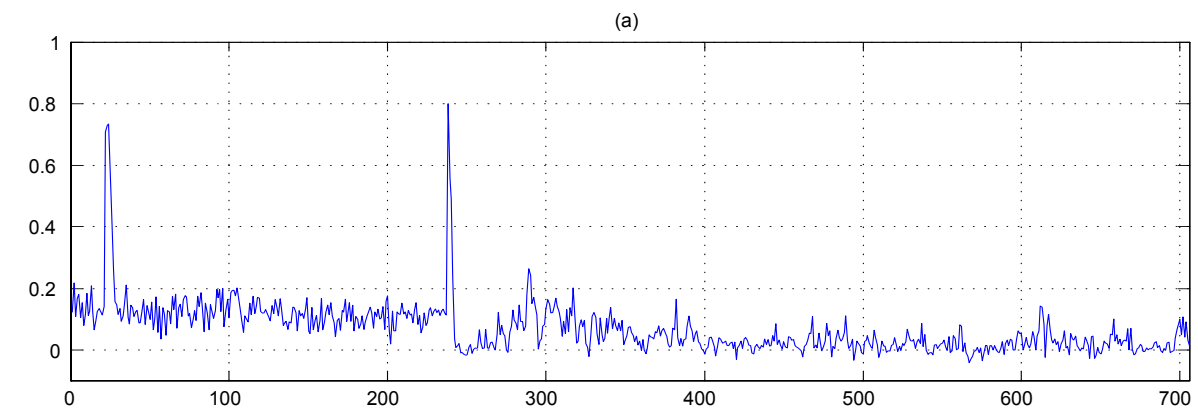

(b)
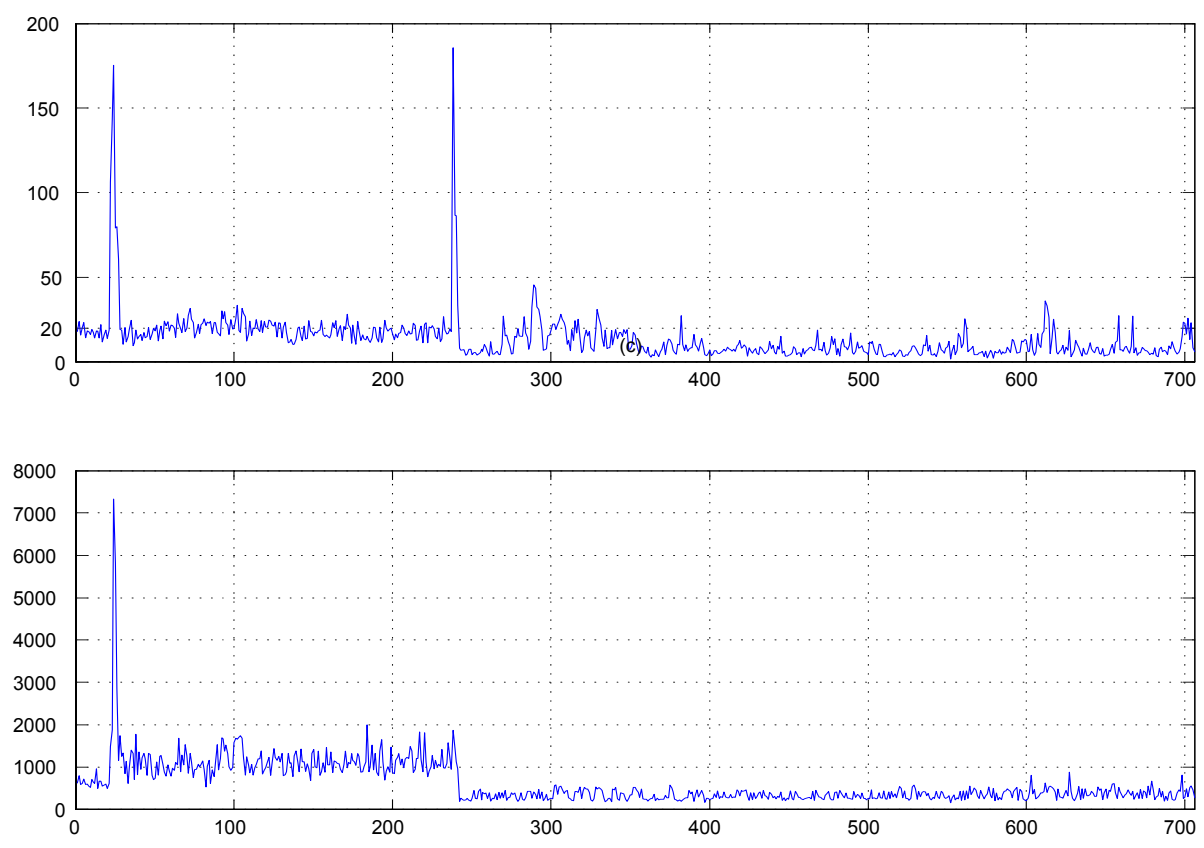

Figure 9: Convergence diagnostics for hyperparameters and volatilities. (a) 20th order sample autocorrelation, (b) inefficiency factors, (c) Raftery and Lewis' total number of runs. See appendix B for details. 